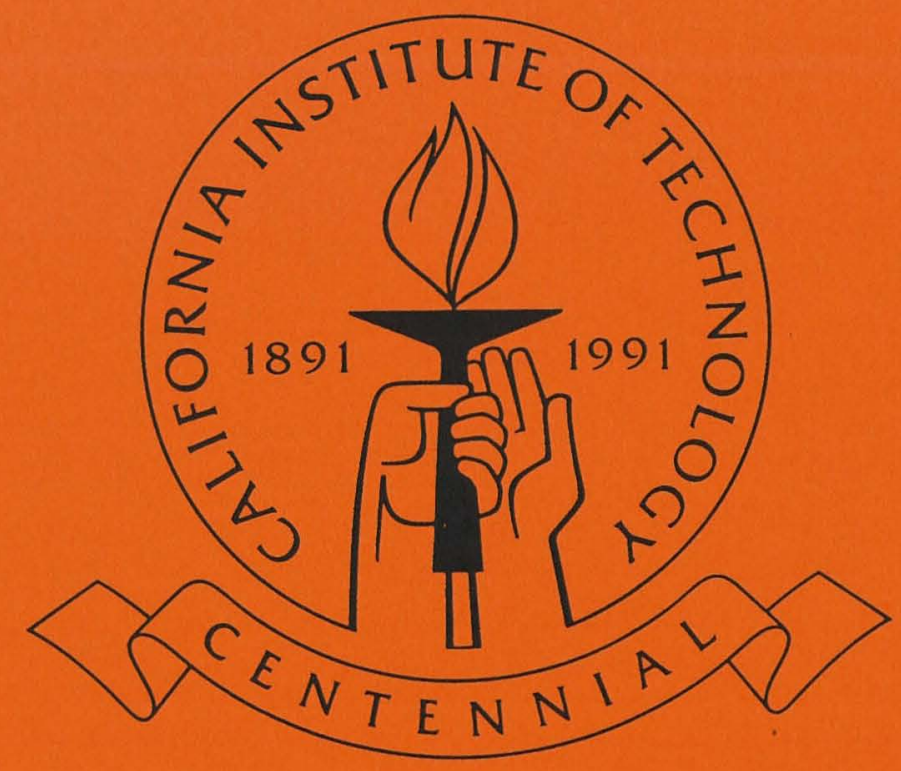

A Primer for Program Composition Notation

\author{
K. Mani Chandy \\ and \\ Stephen Taylor
}

Computer Science Department California Institute of Technology

Caltech-CS-TR-90-10

(Revised 12 December 1990) 


\title{
A Primer for \\ Program Composition Notation
}

\author{
K. Mani Chandy, Stephen Taylor * \\ California Institute of Technology
}

20 June 1990

\section{Abstract}

This primer describes a notation for program composition. Program composition is putting programs together to get larger ones. PCN (Program Composition Notation) is a programming language that allows programmers to compose programs so that composed programs execute efficiently on uniprocessors, distributed-memory multicomputers or shared-memory multiprocessors.

The programs that are put together using PCN can be in PCN itself or in $\mathrm{C}$ or in Fortran. Later implementations of PCN will allow composition of programs in notations in addition to $\mathrm{C}$ and Fortran.

PCN is implemented on a variety of sequential and concurrent architectures including networks of UNIX-based workstations (Sun and NeXT), Symult 2010, Intel iPSC, BBN Butterfly, and Sequent Symmetry.

Several programming examples are presented in the primer. The examples are presented with methods for reasoning about the correctness of PCN programs.

*Supported by NSF under Cooperative Agreement CCR-8809615, and AFOSR and ONR under Grant N00014-89-J-3201. The government has certain rights in this material. 


\section{Overview}

PCN is based on UNITY [3], a theory and a notation for concurrent programming, and on Strand[6]. Composition in PCN is motivated by, but is different from, composition in CSP [8] The motivation for $\mathrm{PCN}$ and a comparison of $\mathrm{PCN}$ with other notations is found in [4]. A programming environment and the run-time support system for $\mathrm{PCN}$ are described in [2] and [7], respectively.

\subsection{New Concepts}

PCN has a three concepts that are not in languages such as $\mathrm{C}$ or Fortran. Next, these concepts are discussed very briefly and informally. Readers may want to skim through sections describing familiar material so as to spend more time on the new concepts.

1. Mutables and Definition Variables: $\mathrm{PCN}$ has two kinds of variables: mutables and definition variables. Mutables are variables as in $\mathrm{C}$. Definition variables are different from variables in $\mathrm{C}$; values of definition variables can be algebraic formulae (such as $y+z$ ), the initial value of a definition variable is a special symbol indicating that it is undefined, and a definition variable is defined at most once. For most programmers, the concept of mutable variables is familiar and the concept of definition variables is new.

2. Composition Operators: A program in PCN is a program heading (program name and arguments), a declaration of types of mutables and a block. A block is an elementary block or a composed block. An elementary block is an assignment statement (similar to assignments in $\mathrm{C}$ or Fortran), or a definition statement that defines definition variables, or a call to a program written in PCN, C, or Fortran. Later implementations will allow composition of programs in other languages. A composed block is a composition operator followed by a list of blocks or guarded-blocks (a block preceded by a boolean expression); the composition operator specifies how the blocks are to be put together.

The only things programmers can do in PCN are: 
- put blocks together using composition operators, or

- define elementary blocks.

PCN has three composition operators: sequential composition, choice composition and parallel composition.

Sequential composition of a list of blocks executes the blocks in sequence, just as in $\mathrm{C}$ or Fortran. Choice composition is an extension of if-thenelse and guarded commands. In a parallel composition of a list of blocks, all blocks in the list are executed in parallel, and the parallel composition completes execution when all its constituent blocks complete execution. For most programmers, sequential and choice composition are familiar concepts, while parallel composition is new.

The central concept of PCN is that of composition - putting blocks together - and once that is mastered all forms of composition are equally easy.

3. Tuples: PCN has a data type called a tuple which is a sequence of items between braces ' $\{$ ' and ' $\}$ '. Linear lists, circular lists, trees and other such linked structures are constructed using tuples.

The concepts of mutables, definition variables and composition are different from concepts found in conventional notations, and therefore, readers should focus attention on these ideas.

\subsection{Highlighting Examples, Syntax and Operation}

Most of this primer consists of examples; a large number are found in the Simple Programming Examples section. There are times when readers will want to study examples carefully and there are other times when readers will want to skip examples. To help identify examples, an example is placed between lines as in: 


\section{Examples of tuples}

The empty tuple: \{\} .

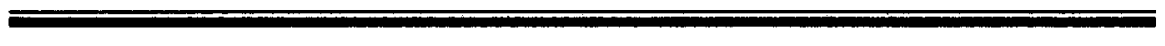

Most examples are on odd-numbered pages, and are therefore on right-hand side pages with most text on left-hand side pages.

For ease in indentification, syntax is placed between lines as in:

$$
\text { tuple } \quad::\{\prec \text { term } \succ\} \mid \ldots
$$

The operation of statements in PCN are described in terms of operations in familiar languages such as C. Operational descriptions of PCN statements are placed between lines as in:

repeat skip until $r h s$ is reducible; assign the reduced value of $r h s$ to $m$. 


\section{Syntax}

In this document we use a stylized syntax to convey the central concepts; a complete formal $\mathrm{BNF}$ is given in Appendix A. All nonterminal symbols are in italics, and all terminal symbols are in plain type. The notation $\prec s u \succ$, where $s u$ is a syntactic unit, represents a list of zero or more instances of the syntactic unit, with multiple instances separated by commas. The notation $\prec s u \succ^{(1)}$ is a list of one or more instances of $s u$ separated by commas. The notation $\langle s u\rangle$ denotes an optional syntactic unit $s u$.

Variable names, comparison operators, macros, and file inclusion are as in C. A comment begins with $/ *$ and ends with $* /$ as in C. Expressions in PCN have the same syntax as arithmetic expressions in $\mathrm{C}$, except that the only operators in $\mathrm{PCN}$ are $\%,+,-, *$ and $/$.

A variable name is a sequence of characters where the first character is a letter, and a character is a letter or a digit. A letter is an upper case or lower case letter of the alphabet, or it is the symbol "..". An upper case letter is different from a lower case letter. 


\section{Data Types}

\subsection{Conventional Data Types}

Conventional data types, such as in $\mathrm{C}$, are also data types in PCN:

1. char for character,

2. int for integer,

3. double for double-precision floating point number.

PCN has one-dimentional arrays of these data types. Arrays in PCN and $\mathrm{C}$ are treated in the same way and are indexed from zero.

Strings in PCN are treated in exactly the same way as in C. A string $S$ is an array $A$ of char, where the characters of $S$ are $A[i]$, in increasing order of $i$ starting with $i=0$ and ending with $i=k$, where $k$ is the smallest index such that $A[k+1]$ is the null character 10 . If $A[0]$ is the null character, $S$ is the empty string. A constant string can be denoted by placing the characters of the string between quotes; for example "PCN" is a string consisting of the three characters: $\mathrm{P}, \mathrm{C}$ and $\mathrm{N}$. The empty string is " ".

In this document, a number is an integer or a double-precision floating point value. We define a simple-value as a number or a character or an array of numbers or a string.

Qualifiers for int in C (such as short, long, and unsigned) are not available in PCN, nor are single-precision floating point numbers and structures.

\subsection{Tuples and Lists}

A tuple is a pointer to a possibly empty sequence of terms, where a term is a simple-value, an expression, or a tuple. A tuple is represented in a program as a sequence of terms between braces - ' $\{$ ' and ' $\}$ ' - where terms are separated by commas.

A list is a special case of tuple. A list is:

1. The empty tuple, \{\} , or 


\section{Examples of tuples}

The empty tuple: \{\} .

A 1-tuple: $\{\{x\}\}$, where the single element of the tuple is itself a 1 -tuple, $\{x\}$.

A 2-tuple: $\{" m s g ", 3\}$.

The empty list: \{\}

A single-element list: $\{d,\{\}\}$ is a list containing a single element, $d$.

A four-element list: $\{a,\{b,\{c,\{d,\{\}\}\}\}\}$ is a list containing the sequence of 4 elements, $a, b, c$ and $d$, in that order. 
2. A 2-tuple, $\{a, b\}$, where the second element of the tuple, $b$, is a list.

PCN has a more succinct notation for lists: a list consisting of a sequence of zero or more elements can be represented by the sequence of elements between the enclosing brackets'[' and ']'. Also, for brevity, we can employ the notation, $\left[L_{1}, L_{2}, \ldots, L_{k} \mid z\right]$, to represent the tuple, $\left\{L_{1},\left\{L_{2},\left\{\ldots\left\{L_{k}, z\right\} \ldots\right\}\right\}\right\}$.

For convenience, the notation $h\left(x_{0}, \ldots, x_{k}\right)$, where $h$ is an identifier and $x_{0}, \ldots, x_{k}$ are tuple-elements, denotes the tuple $\left\{" h ", x_{0}, \ldots, x_{k}\right\}$; this allows programs to be represented as data.

Syntax of Tuples A tuple has the following syntax:

$$
\begin{array}{ll}
\text { tuple } \quad: & \{\prec \text { term } \succ\} \mid \\
& {[\prec \text { term } \succ] \mid} \\
& {[\prec \text { term } \succ(1) \text { ' } \mid \text { term }] \mid} \\
& \text { identifier }(\prec \text { term } \succ) \\
& \\
\text { term } & :: \text { simple-value | expression | tuple }
\end{array}
$$




\section{Examples of list notation}

The empty list: The empty list is []; hence [] $=\{\}$.

A single-element list: $[d]$ is a list containing a single element, $d$; hence $[d]=\{d,\{\}\}$.

A four-element list: $[a, b, c, d]$ is a list containing the sequence of 4 elements, $a, b, c$ and $d$, in that order; hence $[a, b, c, d]=\{a,\{b,\{c,\{d,\{\}\}\}\}\}$.

Catenation of lists: A list, $y$, consisting of a sequence of values, $u, v, w$, followed by another list, $z$, is represented by: $[u, v, w \mid z]$; thus, if $z=[u, c, d]$, and $y=[u, v, w \mid z]$, then $y=[u, v, w, b, c, d]$.

\section{More examples of tuples}

$g(x)$ and $\left\{" g^{\prime \prime}, x\right\}$ denote the same tuple.

$f(x, y)$ and $\{" f ", x, y\}$ denote the same tuple. 
The length Function PCN inciudes a function length which has a singie argument and returns:

- the number of elements in the argument if its argument is a tuple or an array, and

- 1 (one) if its argument is a single number or character.

The argument of length must be a variable. Note the difference between the length function in $\mathrm{PCN}$ and the sizeof function in $\mathrm{C}$ : in $\mathrm{C}$ the function returns the size of its argument in bytes, whereas in $\mathrm{PCN}$ the function returns the number of elements.

Elements of a tuple are referenced in the same way as elements of an array in $\mathrm{C}: t[i]$ is element $i$ of a tuple $t$, for $0 \leq i<$ length $(t)$. 


\section{Examples of length function}

Arrays of numbers:

$l^{*}$ declare $u$ to be an array of 10 doubles */

double $u[10]$;

length $(u)$ is 10 .

A single number:

$I^{*}$ declare $i$ to be an integer */

int $i$;

length $(i)$ is 1 .

A tuple: Let $z$ be the tuple $\{x, y\}$; then length $(z)$ is 2 .

A string:

$1^{*}$ declare $D$ to be an array of 10 chars $* /$

char $D[10]$;

length $(D)$ is 10 . 


\section{Variables}

\subsection{Values of Variables}

At each point in a computation, a variable has precisely one value. The value of a variable is a term. The value of a variable in PCN can be an expression such as $y+z$. In $\mathrm{C}$, execution of the assignment $x=y+z$ causes the value of $x$ to become the value of $y$ plus the value of $z$, and thus the values of variables $x, y$ and $z$ in $\mathrm{C}$ are always numbers; the execution of the assignment in $\mathrm{C}$ does not make the value of $x$ become the formula $y+z$. Indeed, in most notations, values of variables are numbers or characters, but not expressions. Variables in PCN can have expressions as values which allows for a degree of symbolic computation in addition to the usual numeric computation of $\mathrm{C}$ and Fortran.

Notation for Value of a Variable In $\mathrm{C}, x=2$ denotes that $x$ has value 2. We need additional notation to denote the value of a variable in PCN as illustrated by the following example. In PCN, $u=x+y+z$ does not necessarily imply that the value of $u$ is the expression $x+y+z$, because it is possible that the value of $u$ is the expression $v+z$, and the value of $v$ is the expression $x+y$. To avoid this ambiguity, we denote the value of a variable $x$ by value $(x)$. Instead of writing, $x=2$, to denote that $x$ has value 2 , we shall say that value $(x)$ is 2 . Thus, value is a function that maps from variables to terms.

At any point in a computation, we can substitute the value of $x$ for $x$. Therefore, if $u$ has value $v+z$ and $v$ has value $x+y$ at some point in a computation, then $u=v+z$ and $v=x+y$ and hence, $u=(x+y)+z$ at that point. If $u$ and $v$ are undefined, we are not permitted to conclude anything about the relationship between $u$ and $v$; in particular we cannot conclude that $u=v$.

Classes of Variables A variable in PCN is either a mutable or a definition variable. Informally, a mutable is similar to variables in $\mathrm{C}$ and a definition variable is a variable that is assigned at most once. 


\section{Examples of Values of Variables}

Possible values of a variable $x$ are presented next.

Simple-Value: value $(x)$ is 2 .

Expression as Value: value $(x)$ is $y+z$.

Expression as Value: value $(x)$ is $u+v *(y+z)$.

Tuple as Value: value $(x)$ is $\{y\}$.

Tuple as Value: value $(x)$ is $\left\{2, " A^{\prime \prime}, y, z\right\}$. 


\subsection{Mutables}

The type of a mutable is declared in programs in which it is used, its initial value is an arbitrary value of its declared type, and its value can be changed arbitrarily often during a computation by execution of assignment statements that assign values to it. Type declarations are as in C. A mutable type is a $\mathrm{C}$ type (i.e., char, int, or double).

\subsection{Definition Variables}

A definition variable is different from variables used in C. A definition variable is either undefined or defined; it can be defined at most once in a computation. A definition variable is defined to be a term by executing a definition statement in which the definition variable appears on the left-hand side; definition statements are described later.

The value of an undefined definition variable is undefined. The value of a defined definition variable is its definition. The value of a definition variable does not reference mutables.

Definition variables are not declared.

\subsection{Review of Differences between Definition Variables and Mutables}

Declaration Mutables are declared. Definition variables are not declared.

Initial Value The initial value of a mutable is an arbitrary value of its declared type. The initial value of a definition variable is a special symbol indicating that it is undefined.

Expressions as Values The value of a mutable cannot be an expression. The value of a definition variable can be an expression.

Tuples as Values The elements of a tuple are initially definitions variables and can be defined using definition statements. The value of a definition variable can be a tuple; however, no element of a tuple can be a mutable. 
Changes in Values The value of a mutable can be changed arbitrarily many times. The value of a definition variable can be changed at most once, from undefined to a defined value. Once a definition variable is defined, its value remains unchanged forever thereafter. 


\section{Programs}

A program consists of a heading followed by a declaration section followed by a block. The heading is the program name and a list of formal parameters, as in C. In PCN all parameters are passed by reference, unlike in in $\mathrm{C}$ where parameters can be passed by value. The syntax of a declaration section is identical to that in $\mathrm{C}$. The scope of a variable is the program in which it appears: all variables that appear in a program are either formal parameters or local variables of the program. All mutables referenced in a program are declared in the declaration section of the program; definition variables are not declared.

Local variables in PCN are local to the program in which they are declared, whereas local variables in $\mathrm{C}$ can be declared to be local to blocks within programs. Also, $\mathrm{C}$ allows programs to access variables that are not formal parameters or local variables of the program, whereas PCN does not.

The dimensions of a local array of a program can change from one call of the program to the next, unlike in $\mathrm{C}$ where some of the dimensions of a multidimensional local array must remain unchanged in all calls.

The syntax of a block is:

\begin{tabular}{ll}
\hline block & $:$ elementary-block | composed-block \\
elementary-block & $:: \begin{array}{l}\text { definition-statement } \mid \\
\text { assignment-statement } \\
\text { program-call }\end{array}$ \\
\hline
\end{tabular}

Composed blocks are discussed later; the next few sections discuss each of the forms of elementary blocks. 
An Example of Formal Parameters and Local Variables

$$
\begin{aligned}
& p(\text { sum }, x) \\
& \text { int sum, } \\
& \{? \quad x ?=[m \mid x s] \rightarrow \\
& \} \quad\{; v:=m, \text { sum }:=\text { sum }+v, p(\text { sum }, x s)\} \\
& \} \quad
\end{aligned}
$$

The operators in this example are not important here; only the heading and the declarations are relevant. The first line is the heading for a program with name, $p$, and two formal parameters, sum and $x$. The second line declares sum and $v$ to be integer. Therefore, sum and $v$ are mutable. Since the types of $x, x s$ and $m$ are not declared, they are definition variables. Since $x s$, $m$ and $v$ are not formal parameters, they are local variables of program $p$. 


\section{Definition statements}

A definition statement has the following syntax:

$$
\text { definition-statement } \quad:: \quad \text { definition-variable }=\text { term }
$$

The execution of the definition statement $x=r h s$ completes, and at completion, value $(x)$ is $r h s^{\prime}$ where $r h s^{\prime}$ is obtained by substituting value(v) for each mutable $v$ in $r h s$. Mutables do not appear in $r h s^{\prime}$, and hence the value of a definition variable does not name mutables.

The value of an expression in PCN can be an array. For example if mutable $m$ is declared to be an array of integers, then the value of the expression ' $m$ ' is an array. Hence, upon completion of the execution of the statement $x=m$, the value of $x$ is an array.

Arithmetic operators in PCN are identical to those in C, and hence their operands are numbers, not arrays of numbers. So the expression ' $m+1$ ', where $m$ is an array, is incorrect. 
Examples of Definition Statements Without Mutables

In these examples, $z$ is a definition variable.

number: Execution of the definition statement

$$
z=3.0
$$

terminates with

$$
\operatorname{value}(z)=3.0
$$

string: Execution of the definition statement,

$$
z=\text { "abc" }
$$

terminates with

$$
\operatorname{value}(z)=\text { "abc". }
$$


Examples of Definition Statements With Mutables

In these examples, $z$ and $y$ are definition variables, and $m$ is a mutable with value 2 at the point in the computation at which the definition statements are executed.

expression: Execution of the definition statement

$$
z=y+m+5
$$

terminates with

$$
\operatorname{value}(z)=y+2+5
$$

tuple: Execution of the definition statement

terminates with

$$
z=\{y,\{m\}, 5\}
$$

$$
\operatorname{value}(z)=\{y,\{2\}, 5\} \text {. }
$$

tuple: Execution of the definition statement

$$
z=[m, " b "]
$$

terminates with

$$
\operatorname{value}(z)=\left[2, " b^{\prime \prime}\right] \text {. }
$$




\section{Examples of Definition Statements With Arrays}

If $A$ is a 2 -element integer array with $A[0]=1$, and $A[1]=2$, then the definition statement, $z=A$ terminates with $z$ defined as a 2 -element integer array with value $(z[0])=1$, and $\operatorname{value}(z[1])=2$. 


\section{$7 \quad$ Reducibility}

The meaning of an assignment is based on the concept of reducibility. For a term $e$, the reduced value of $e$ is a simple-value or tuple $x$, where $x=e$. A term $e$ is reducible at a point $t$ in a computation if and only if a reduced value of $e$ can be computed at $t$, i.e.,

1. $e$ is a simple-value or a tuple, in which case the reduced value of $e$ is $e$, or

2. value $(e)$ is $f$ and $f$ is reducible, in which case the reduced value of $e$ is the reduced value of $f$, or

3. $e$ is an expression and all variables in $e$ are reducible, in which case the reduced value of $e$ is computed by substituting the reduced value of $v$ for each variable $v$ in $e$ and evaluating.

\subsection{Properties of Reducibility}

Value Equals Reduced Value For all variables $x$, the value of $x$ is equal to its reduced value. This is because the reduced value of $x$ is obtained from the value of $x$ by substitution and expression evaluation.

Unique Reduced Values The reduced value of an element $e$ at a point in a computation is unique. For example, if the reduced value of $e$ is 2 at a point in a computation then the reduced value of $e$ cannot also be 3 at that point.

Mutables Are Reducible A mutable is reducible at all points in computation and the reduced value of a mutable is its value.

Undefined Definition Variables Are Not Reducible An undefined definition variable is not reducible because the value of an undefined definition variable is a special symbol indicating that it is undefined, and hence none of the rules of reducibility can be employed to compute a simple value or tuple for it. 


\section{Examples of Reducibility}

\section{Example 1}

Let $z$ be a definition variable, and let $m$ be a mutable that is declared to be an integer. If at a point $t$ in a computation, value $(z)$ is 1 and value $(m)$ is 2 , then $z+m$ is reducible and its reduced value is 3 , at $t$.

If $m=4$ at a later point $t^{\prime}$, then the reduced value of $z+m$ is 5 at $t^{\prime}$.

\section{Exampie 2}

If at a point $t$ in a computation, $y$ is undefined, then $y$ is not reducible at $t$. If at $t$, the value of $z$ is $y+1$, then $z$ is not reducible at $t$ because $y$ is not reducible at $t$.

\section{Example 3}

If at a point $t$ in a computation, value $(z)$ is $y+1$, and value $(y)$ is 0 , then $z$ is reducible and its reduced value is 1 at $t$. Furthermore, if $z$ is a definition variable, the reduced value of $z$ remains 1 at all points after $t$. 
Defined Definition Variables Á defined definition variable may or may not be reducible. For example, if value $(x)=y+z$, where $x, y$ and $z$ are definition variables, then $x$ is not reducible if $y$ or $z$ is not reducible. If, however, $y$ and $z$ are reducible with reduced values (say) 1 and 2 respectively, then $x$ is reducible and has reduced value 3 .

Once Reducible, Remains Reducible Once a term is reducible it remains reducible forever thereafter. The reasons for this are as follows. Mutables, simple-values and tuples are always reducible. Once a definition variable is reducible it remains reducible because its value remains unchanged. Once an expression (that can name mutables and definition variables or both) is reducible it remains reducible.

Reduced Values of Definition Variables The reduced value of a definition variable remains unchanged. For example, if the reduced value of $z$ is 2 at some point in a computation, then the reduced value of $z$ remains 2 forever thereafter. Likewise, the reduced value of an expression, that does not name mutables, remains unchanged. For example, it the reduced vaiue of $y+z$ is 3 at some point in a computation (where $y$ and $z$ are definition variables), then the reduced value of $y+z$ remains 3 thereafter.

Reduced Values of Mutables The reduced value of a mutable can change; for instance mutable $m$ can have value 2 at some point in a computation and value 3 at a later point. Likewise, the reduced value of an expression that names mutables can change. For example, the value of expression $y+z+m$ can change, where $m$ is a mutable, because $m$ can change value. 
More Examples of Reducibility

\section{Example 4}

If at a point $t$ in a computation, $\operatorname{value}(y)=\{1, z\}$, then $y$ is reducible and its reduced value is $\{1, z\}$ at $t$. Note that $y$ is reducible even if $z$ is not reducible.

\section{Example 5}

If at a point $t$ in a computation, value $(y)=A$, where $A$ is an integer array, then $y$ is reducibie, and its reduced value is $A$ at $t$.

\section{Example 6}

If at a point $t$ in a computation, value $(y)=x+1$, and value $(x)=$ $2 * y-2$, then both $x$ and $y$ are nonreducible at $t$. (Note that from the mathematics of simultaneous equations we can conclude $x=$ 0 and $y=1$, but according to our definitions $x$ and $y$ are not reducible.) 


\section{Assignment Statements}

The syntax of an assignment statement is:

$$
\text { assignment-statement :: mutable := expression }
$$

The execution of the assignment statement $m:=r h s$ where $r h s$ is an expression and $m$ is a mutable variable, declared to be one of the types in $\mathrm{C}$, is as follows:

repeat skip until $r h s$ is reducible;

assign the reduced value of $r h s$ to $m$.

A skip is an operation that does nothing, and it is sometimes referred to as a 'no-op.' Therefore, while $r h s$ is not reducible, the program executes operations that do nothing - in other words, the program waits. When $r h s$ becomes reducible, the reduced value of $r h s$ (coerced to be the same type as $m$ ) is assigned to $m$, and the assignment completes. If $r h s$ never becomes reducible the assignment does not complete, and execution of the assignment statement is an infinite number of skips. Note that if $r h s$ does not reference definition variables, the assignment statement is executed without skips, because rhs is reducible; in this case the assignment is executed in the same way as assignments in $\mathrm{C}$. 
Simple Examples of Assignment

Right-Hand Side Does Not Reference Definition

Variables

int $m, i, j, u[10], v[10]$

$\ldots, m:=i+j, \ldots, u:=v, \ldots$

The assignment $m:=i+j$ is executed in the same way as the assignment $m=i+j$ in $\mathrm{C}$ : the sum of the values of the integers $i$ and $j$ is assigned to $m$.

The assignment $u:=v$ makes $u$ become the array $v$.

Right-Hand Side References Definition Variables

int $m$;

$\ldots, m:=z, \ldots$

The assignment, $m:=z$, where $m$ is an integer mutable, and $z$ is a definition variable, is executed as follows. While $z$ is not reducible, skip. When $z$ becomes reducible, assign its value (coerced to integer) to $m$. If $z$ never becomes reducible, execution of the assignment does not complete. 


\section{Program Calls}

The syntax of a program call is:

$$
\begin{aligned}
\text { program-call }: & \text { program-name }(\prec \text { term } \succ) \mid \\
& \text { 'definition-variable }(\prec \text { term } \succ)
\end{aligned}
$$

A program call program-name $(\prec t e r m \succ)$ is the same as a function call in $C$, except that all parameter passing is by reference, and the program does not return a value (in the way that a function does). Later, we will describe two modifications to this syntax that allow programmers to specify processors on which called programs are to be spawned, and modules (files) that contain the source texts of the called programs; these do not change the essential meaning of program calls.

\subsection{Calling C programs from PCN}

PCN programs can call $\mathrm{C}$ programs. The actual parameters in a call to a $\mathrm{C}$ program can be definition variables or mutables. All parameter passing in PCN is by reference; hence, the arguments of the $\mathrm{C}$ program must be pointers to simple-values (i.e., char, int, or double). The execution of a $\mathrm{C}$ program call is as follows:

repeat skip until all actual parameters are reducible; execute the C program.

We do not specify the behavior of $\mathrm{PCN}$ programs that call $\mathrm{C}$ programs which continue execution for ever; therefore, programmers must ensure that C programs terminate. 


\section{Examples of Calls to Programs}

Consider the $\mathrm{C}$ program:

$q(v, w, x)$

int $* v, * w, * x$;

$\{* v=* v-* x ; * w=* w+* x\}$

The execution of the call $q(a, b, z)$, where $a$ and $b$ are mutable integer variables and $z$ is a definition variable, is as follows: repeat skip until $z$ is reducible; when $z$ becomes reducible, execute $q\left(v, w, z^{\prime}\right)$ where $z^{\prime}$ is the reduced value of $z$. Note that even though $z$ is a definition variable, and its value cannot be changed by the $\mathrm{C}$ program, $z$ is passed by reference and not by value. That is why the type declaration of formal parameter, $x$, is 'int $* x$ ', and not 'int $x$ '. Definition variable $z$ must reduce to an integer value because the corresponding formal parameter $x$ is a pointer to an integer.

Consider the PCN program:

$p(w, x, y, z)$

$\{\| y=w+x, \quad z=w-x\}$

A call $p(a, b, c, d)$ of program $p$ causes program $p$ to be executed, even if actual parameters are not reducible. We will see later that the program completes, and at completion value $(c)$ is $a+b$ and value $(d)$ is $a-b$, regardless of whether $a$ or $b$ are reducible. 


\subsection{Calling Fortran Programs from PCN}

Fortran programs are called in the same way as $\mathrm{C}$ programs. Parameter passing in Fortran is by reference, as it is in PCN.

\subsection{Calling PCN Programs from PCN}

PCN programs can call PCN programs; the actual parameters of the call can be mutables or definition variables. The called program is initiated even if some or all of the actual parameters are not reducible.

There is a difference between the execution of calls to $\mathrm{C}$ programs and PCN programs. A called $\mathrm{C}$ program is initiated only when all its actual parameters are reducible. A called PCN program is initiated without waiting for all its actual parameters to be reducible. (A strict semantic is used for $\mathrm{C}$ calls and a nonstrict semantic for PCN calls.)

Types of actual parameters should be the same as types of corresponding formal parameters in calls to PCN programs. In particular, an actual parameter should be a definition variable if and only if the corresponding formal parameter is a definition variable.

\subsection{Program Names as Actual Parameters of Programs}

A program-name can be passed as an argument of a program. To distinguish a variable whose value is a program name from a program name, the symbol ' is employed. For example $y(x)$ is a call of program $y$, whereas ' $y(x)$ is a call to a program $g$, where the reduced value of $y$ is " $g$ ".

Recall that $f\left(x_{0}, \ldots, x_{k}\right)$ represents the tuple $\left\{" f ", x_{0}, \ldots, x_{k}\right\}$. Similarly, ' $f\left(x_{0}, \ldots, x_{k}\right)$ represents the tuple $\left\{f, x_{0}, \ldots, x_{k}\right\}$.

The program call ' $y(x)$ where $x$ is a list of actual parameters and $y$ is a definition variable, is executed as follows:

repeat skip until $y$ is reducible; execute $g(x)$ where " $g$ " is the reduced value of $y$. 


\section{Example of a Program Name as A Parameter}

Next, consider a program map, which has a formal parameter operator defined as a string that is a name of a program.

map(operator, lst, result)

int result;

$\{$ ? lst $?=[$ head $\mid$ tail $] \rightarrow$

$\{;$ 'operator(head, result),

\}

map(operator, tail, result)

\}

Passing a Program Name as a Parameter

Calling the preceding program with $\operatorname{map}(" a d d ", L, R)$, causes the following block to be executed:

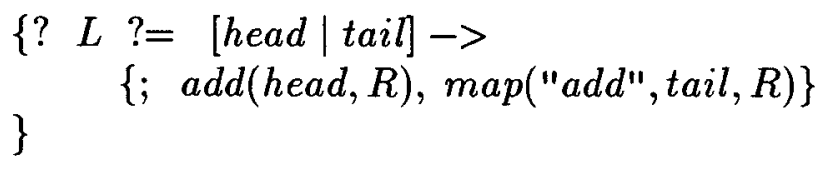




\section{Composed Blocks}

A composed block has the following syntax.

$$
\begin{aligned}
\text { composed-block :: } & \left\{; \prec \text { block } \succ^{(1)}\right\} \mid \\
& \left\{\| \prec \text { block } \succ^{(1)}\right\} \mid \\
& \left\{? \prec \text { guard } \rightarrow \text { block } \succ^{(1)}\right\}
\end{aligned}
$$

where ';' denotes sequential composition, ' $\|$ ' denotes parallel composition, and '?' denotes choice composition.

\section{Sequential Composition}

Let $d$ be the block $\left\{; b_{1}, \ldots, b_{k}\right\}$, where $k>0$. The execution of $d$ is a sequential execution of $b_{i}$, in order, from $i=1$ to $i=k$.

\section{Parallel Composition}

Let $d$ be the block $\left\{\| b_{1}, \ldots, b_{k}\right\}$, where $k>0$, and for all $i$ where $0<i \leq k$, $b_{i}$ is a block. In an execution of $d$, all blocks $b_{i}$ are executed in parallel. Block $d$ terminates when the computations of $b_{i}$ terminate for all $i$. (A computation of $d$ is a fair interleaving of computations of $b_{i}$, for all $i, 0<i \leq k$. Execution is fair in the following sense: For all $i$, it is always the case that eventually computation of $b_{i}$ will progress if $b_{i}$ has not terminated.)

Programmers must ensure that the following condition about shared variables is satisfied.

Restriction on Shared Variables in Parallel Composition Shared mutables must not change value during parallel composition. 


\section{Example of Sequential Composition}

$p(j, k, x, y)$

int $j, k, m$;

$/^{*}$ Let the value of $j$ be $J . * /$

$/^{*} m$ is a local integer mutable of $p^{*} /$

\{

$m:=2$,

$l^{*} \operatorname{value}(m)$ is $2^{*} /$

$x=m+1$,

$l^{*}$ The reduced value of $x$ is 3 . */

$k:=y+j$

$/^{*}$ value $(k)$ is sum of the ${ }^{*} /$

\}

$l^{*}$ reduced values of $y$ and $j . * /$

First $m$ becomes 2, then $x$ is defined as $2+1$ (and hence its reduced value is 3 ), and then, after executing skips until $y$ becomes reducible, $x$ becomes the sum of the values of $y$ and $j$. 
This restriction is equivalent to: In a parallel composition block $d$ defined as $\left\{\| b_{1}, \ldots, b_{k}\right\}$,

for each variable $v$ accessed in distinct blocks $b_{i}$ and $b_{j}$ :

1. $v$ remains unchanged during the execution of $d$, or

2. $v$ is a definition variable.

For this purpose, each element of a shared array is treated as separate variable; therefore, blocks composed in parallel can modify a shared array, but each element of the array must remain unchanged or be accessed by at most one block. Similarly, each element of a tuple is treated as a separate element.

An important consequence of this restriction is that blocks, composed in parallel, interact with each other in a disciplined manner. Consider a predicate $z$ that references only variables that appear in one of the constituent blocks, say $b_{i}$ of parallel composition block $d$. For example, $z$ could be $u=v+1$, where $u$ and $v$ are variables referenced in block $b_{i}$. Suppose we can reason from the text of block $b_{i}$ (i.e., by considering block $b_{i}$ in isolation, independent of the blocks that $b_{i}$ is composed with) that predicate $z$ holds at some point $p$ in $b_{i}$. In our reasoning we are not permitted to conclude that definition variables are undefined if they are undefined in $b_{i}$ (because definition variables can be defined in blocks that are composed with $b_{i}$ ). Then, no matter what blocks are composed in parallel with $b_{i}$, we are assured that our reasoning is valid, and $z$ holds at $p$.

An equally important consequence of this restriction is that we do not have to be concerned about atomicity in parallel composition. 
Example of Parallel Composition

$\{\| p(j, k, x, y), y=x+j\}$

(Program $p$ is defined in the previous example.) This parallel composition block obeys our convention about shared variables. The only shared mutable is $j$ which is not modified in the block. Let the value of $j$ be 1 when this block is executed. The computation of this block terminates, and at termination, the reduced value of $x$ is 3 , and $y$ is 4 , and $k$ is 5 .

A possible computation of the parallel composition block is: $y$ is defined as $x+1$ (assuming $j=1$ ), then, in program $p: m$ becomes 2 , then $x$ becomes defined as $2+1$, and finally $k$ becomes 5 . Note that $y$ can become defined before $x$ becomes defined. 


\section{Choice Composition}

A choice composition block is similar to a guarded command [5]. A guard in a choice composition block is a boolean expression or the keyword default. At a point in a computation, a boolean expression is:

1. not reducible, or

2. reducible and has value true, or

3. reducible and has value false.

We shall see later that once a guard is reducible it remains reducible for ever thereafter.

There can be at most one default guard in a choice composition block. A choice block without a default guard is equivalent to a choice block with the addition of the guarded block: default $->$ skip. therefore, we can restrict attention to choice blocks that contain precisely one default guard.

The basic idea about execution of the choice block:

$$
\left\{? \text { default } \rightarrow b_{0}, G_{1} \rightarrow b_{1}, \ldots, G_{k}->b_{k}\right\}
$$

is as follows:

1. If all guards are false then execute $b_{0}$; execution of the choice block terminates when execution of $b_{0}$ terminates.

2. If at least one guard is true then execute any block $b_{i}$ where $G_{i}$ is true; execution of the choice block terminates when execution of $b_{i}$ terminates.

3. Because guards can be nonreducible, there is a third possibility: at least one guard is nonreducible and no guard is true. In this case the program repeatedly executes skips until one of the first two conditions holds. 


\section{Examples of Choice Blocks}

Example 1

Consider the choice-block:

$\{? \quad x>=0 \rightarrow y=x+1$,

$x<=0 \rightarrow y=x-1$

\}

The execution of this block is as follows. While $x$ is irreducible, skip. When $x$ becomes reducible, if $x>0$, then only the first guard holds, and hence $y$ is defined as $x+1$; if $x<0$, then only the second guard holds, and hence $y$ is defined as $x-1$; if $x-0$, then both guards hold, and a nondeterministic choice is made to define $y$ either as $x+1$, or as $x-1$. Execution of the block terminates after $y$ is defined.

Example 2

Consider the choice-block:

$\{? \quad x>=0 \rightarrow y=x+1\}$

The execution of this block is as follows. While $x$ is irreducible, skip. When $x$ becomes reducible: if $x \geq 0$ then $y$ is defined as $x+1$ else $y$ is left unchanged; execution of the block terminates. 


\subsection{Guards}

A guard is either a sequence of one or more guard elements or default. The syntax of a guard is:

$$
\text { guard }:: \prec \text { guard-element } \succ^{(1)} \mid \text { default }
$$

Case 1: If All Guard-Elements are Reducible If all the guard-elements of a guard $G$ are reducible, then $G$ is reducible, and the value of $G$ is a 'conditional-and' of its guard-elements: Each of the guard-elements in the sequence is evaluated in order until all guard-elements in the sequence are evaluated or a guard-element evaluates to false; if all guard-elements evaluate to true the value of the guard is true, otherwise the value of the guard is false. The evaluation of a guard is similar to the evaluation, in C, of an expression consisting of the sequence of guard-elements with the logical connective \&\& between guard-elements.

Case 2: At Least One of the Guard-Elements is Irreducible Next, consider the case where at least one of $G$ 's guard-elements is irreducible: If all guard-elements before the first nonreducible element of $G$ evaluate to true, then $G$ is not reducible; otherwise, $G=$ false.

\subsection{Guard-Elements}

The syntax of a guard-element is:

$$
\begin{array}{r}
\text { guard-element } \begin{array}{r}
:: \text { type-check } \mid \text { comparison } \mid \\
\text { data-check } \mid \text { pattern-match }
\end{array}
\end{array}
$$




\section{Another Example of a Choice Block}

Example 3

Consider the choice-block:

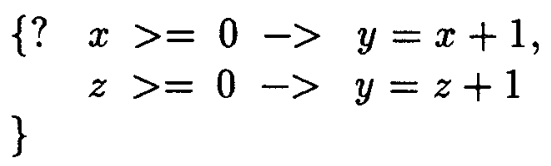

Repeat skips until both guards are reducible and both have reduced value false, or at least one of the guards is reducible and has reduced value true. In the former case the choice block terminates without changing the value of any variable. In the latter case, if both guards have reduced value true then execute either $y=x+1$ or $y=z+1$, if only $x \geq 0$ has reduced value true then execute $y=x+1$, and if only $z \geq 0$ has reduced value true then execute $y=z+1$. 
Type Checks The syntax for type-check is:

$$
\begin{array}{ll}
\text { type-check } & :: \text { type-name(definition-variable) } \\
\text { type-name } & :: \text { int | double | char | tuple }
\end{array}
$$

If $x$ is not reducible, type-check $h(x)$ is not reducible. If $x$ is reducible, type-check $h(x)$ evaluates to:

1. true if the reduced value of $x$ is of type $h$ or is an array of type $h$,

2. false otherwise.

For example, if $x$ is reducible, then int $(x)$ holds if and only if the reduced value of $x$ is an integer or an array of integers.

Comparison The syntax of a comparison is:

$$
\begin{array}{ll}
\text { comparison }:: & \text { term equality-test term } \mid \\
& \text { expression ordering expression } \\
\text { equality-test }::==\mid != \\
\text { ordering }::<|<=|>\mid>=
\end{array}
$$

A comparison $x \alpha y$, where $\alpha$ is an ordering, is reducible if and only if both $x$ and $y$ are reducible; the reduced values of $x$ and $y$ must be numbers or arrays. Numbers and strings contained in character arrays are compared as in $\mathrm{C}$; integer and double arrays are compared consecutively by element.

A comparison $x \alpha y$, where $\alpha$ is an equality-test, is reducible only if both $x$ and $y$ are reducible. An equality-test $x==y$, where the reduced values of 


\section{Example of Type Check and Comparison}

$\{? \operatorname{int}(x), x>=y \rightarrow z=x+1\}$

Consider the case where $y$ is irreducible and $x$ is reducible. If the reduced value of $x$ is an integer, then the guard, is not reducible because the first guard-element evaluates to true and the second guard-element is not reducible. If the reduced value of $x$ is a character, then the guard evaluates to false, because the first guard-element evaluates to false.

Next consider the same program except that the order of guardelements is reversed.

$$
\{? \quad x>=y, \operatorname{int}(x) \rightarrow z=x+1\}
$$

As before, consider the case where $y$ is irreducible and $x$ is reducible. As in the last example, if the reduced value of $x$ is an integer, then the guard, is not reducible because the first guard-element is not reducible. If the reduced value of $x$ is a character then the guard is not reducible for the same reason. Note that in the previous example, if $x$ is a character the guard is reducible. This example shows that the ordering of guard-elements can make a difference to the computation. 
$x$ and $y$ are tuples, is equivalent to the following sequence of equality-tests:

length $(x)==$ length $(y)$, and for all $i$ where $0 \leq i<\operatorname{length}(x): x[i]==y[i]$.

Thus, equality tests of tuples are equivalent to sequences of equality-tests without tuples. A comparison $x==y$ is reducible if $x$ and $y$ reduce to simple values. Equality of characters and numbers is as in $\mathrm{C}$.

Inequality is defined as the negation of equality.

Data Check The syntax of a data-check is:

data-check :: data(definition-variable)

If $x$ is reducible then data $(x)=$ true. If $x$ is not reducible, then data $(x)$ is also not reducible. The value of $\operatorname{data}(x)$ is never false. 


\section{Testing Equality of Tuples}

$$
\{? x==y \rightarrow p(x)\}
$$

In the equality test, $x$ or $y$ can be tuples (and therefore can be lists). If $x=[0,1,2]$, then the equality test succeeds only if $y$ is equal to the same list. If $y$ is $[0,1 \mid z]$, where $z$ is a nonreducible definition variable, then $x==y$ is not reducible.

As another example, consider the case where the reduced value of $x$ is $z$ and the reduced value of $y$ is also $z$. The equality test is reducible if and only if $z$ is reducible. Of course, if $z$ is reducible, the equality test succeeds. 


\subsection{Pattern Matches}

A pattern-match is merely a syntactic convenience for operating on tuples. It has the following syntax:

$$
\begin{array}{ll}
\text { pattern-match } & :: \text { variable } ?=\text { pattern } \\
\text { pattern } & ::\{\prec \text { pattern-element } \succ\} \\
\text { pattern-element } & :: \text { simple-value | definition-variable | pattern }
\end{array}
$$

A pattern-match $x ?=$ pat succeeds (i.e., has reduced value true) if the reduced value of $x$ is a pattern of the same 'form' as the pattern, pat, on the right. For example, if pat is $\{u, v\}$, then the match succeeds if the reduced value of $x$ is a tuple of size 2. If a match succeeds, a variable in the pattern become an alias for the corresponding element of the tuple for the remainder of the guard and its associated block. Thus the pattern-match $x ?=\{u, v\}$ succeeds if $x$ is the 2-tuple $\{x[0], x[1]\}$, and if the match succeeds then $u$ becomes an alias for $x[0]$, and $v$ becomes an alias for $x[1]$ for the remainder of the guard and its associated block. Next, matches are discussed in more detail.

Evaluation of a Pattern-Match A definition variable that appears in a pattern must be undefined when the pattern-match is evaluated. Mutables cannot appear in patterns.

A pattern match, $z ?=$ pat can be transformed into a sequence of guardelements without the pattern match by the following syntactic transformation: Replace the pattern match by,

$$
\operatorname{tuple}(z),(\text { length }(z)==\operatorname{length}(\text { pat }))
$$

and for each $i$, where $0 \leq i<\operatorname{length}(z)$, if pat $[i]$ is a

pattern add the pattern-match, $z[i] ?=\operatorname{pat}[i]$, 


\section{Simple Patterns}

In the following example, $z, h d$ and $t l$ are definition variables that are undefined at the point at which the pattern matches are executed.

$z ?=\{h d, t l\} \quad \rightarrow\{\| h d=u, v=t l\}$

is equivalent to:

$\operatorname{tuple}(z), \operatorname{length}(z)==2 \quad \rightarrow\{\| z[0]=u, v=z[1]\}$

The match $z ?=\{h d, t l\}$ succeeds if $z$ is a tuple of the same form as $\{h d, t l\}$, i.e., if $z$ is a 2-tuple. If the match succeeds, then $h d$ is an alias for $z[0]$, and $t l$ is an alias for $z[1]$ in the remainder of the guard and its associated block. 
simple-value add the equality-test, $z[i]==\operatorname{pat}[i]$,

definition variable replace all instances of pat $[i]$ by $z[i]$ in the remainder of the guard and its associated block. 


\section{Patterns with Strings and Numbers}

$z ?=\{v,\{y\}, 2, " m s g "\} \quad \rightarrow\{\| v=y\}$

is equivalent to

$$
\begin{aligned}
& \operatorname{tuple}(z),(\text { length }(z)==4), \\
& (\text { tuple }(z[1]),(\text { length }(z[1])==1)), \\
& (z[2]==2),(z[3]==" m s g ") \rightarrow \\
& \{\| z[0]=z[1][0]\}
\end{aligned}
$$

The match succeeds if the reduced value of $z$ is the same form as the pattern, i.e., if $z$ 's reduced value is a 4-tuple where $z[3]$ and $z$ [4] are the integer 2 and the string " $m s g$ ", respectively, and where the form of $z[1]$ is the pattern $\{y\}$. If the match succeeds, $v$ and $z[0]$ are aliases of each other, and likewise, $y$ and $z[1][0]$ are aliases of each other.

\section{Patterns with Lists}

$z ?=[w \mid x] \rightarrow\{; s u m:=s u m+w, p(s u m, x)\}$

is equivalent to

$$
\begin{aligned}
& \operatorname{tuple}(z), \text { length }(z)==2, \rightarrow \\
& \quad\{; \text { sum }:=\operatorname{sum}+z[0], p(s u m, z[1])\}
\end{aligned}
$$




\section{Syntactic Sugar: Composition of Guarded Statements}

The syntax that we gave for composed blocks was:

$$
\begin{aligned}
\text { composed-block :: } & \left\{; \prec \text { block } \succ^{(1)}\right\} \mid \\
& \left\{\| \prec \text { block } \succ^{(1)}\right\} \mid \\
& \left\{? \prec \text { guard } \rightarrow \text { block } \succ^{(1)}\right\}
\end{aligned}
$$

Thus parallel and sequential composition compose blocks but not guard $->$ blocks. For notational convenience we relax this syntax to allow parallel and sequential composition of guard $\rightarrow>$ blocks. Each guard $->$ block is transformed into the choice block $\{?$ guard $\rightarrow>$ block $\}$. The sugared syntax is:

$$
\begin{aligned}
& \text { composed-block :: \{; ঝunit } \left.\succ^{(1)}\right\} \mid \\
& \left\{\| \prec \text { unit }^{(1)}\right\} \mid \\
& \left\{? \prec \text { guard } \rightarrow \text { block } \succ^{(1)}\right\} \\
& \text { unit :: block | guard } \rightarrow \text { block }
\end{aligned}
$$

We may find it convenient to think of all composition blocks as consisting of a composition operator operating on a list of guard $\rightarrow$ block, where some guards can be true. 


\section{Examples of Syntactic Sugar}

The following programs, the first with the added sugar, and the second without it, are equivalent:

$$
\begin{aligned}
& f(t, z) \\
& \left\{\begin{array}{l}
t
\end{array} ?=\{\text { left,val,right }\} \quad \rightarrow\{\| f(\text { left }, l), f(\text { right }, r),\right. \\
& l>=r \rightarrow z=1+l \text {, } \\
& r>=l \rightarrow z=1+r \\
& \text { \} } \\
& \} \\
& g(t, z) \\
& \left\{\begin{array}{l}
t
\end{array} ?=\{\text { left, val, right }\} \quad \rightarrow\{\| g(\text { left }, l), g(\text { right }, r),\right. \\
& \{? l>=r \rightarrow z=1+l\} \text {, } \\
& \{? r>=l->z=1+r\} \\
& \text { \} }
\end{aligned}
$$


The Variable Name ',' There are a few places where we would like to refer to a variable that we do not wish to use later. For instance, we may want a pattern with 3 elements, but we wish to use only the first of the 3 elements. Instead of coming up with names for the remaining two elements we can use ' - as a name for both elements. Each instance of '.' is treated as the name of a new unique variable.

\section{Built-In Programs and I/O}

make_tuple One of the built-in programs provided in PCN is make_tuple which has two arguments, $n$ and $x$ where $x$ is a definition variable that is defined by make_tuple and $n$ is a variable unchanged by the program. The program defines $x$ to be a tuple of $n$ elements, where $n$ is a nonnegative integer, where each element of $x$ is a definition variable. (If $n \leq 0$ then $x$ is defined to be the empty tuple.) The elements of tuple $x$ are left undefined by make_tuple. For example, make_tuple $(2, x)$ defines $x$ to be a 2 -tuple, leaving definition variables $x[0]$ and $x[1]$ undefined.

The program call make_tuple $(n, x)$ is executed as follows:

repeat skip until $n$ is reducible;

coerce $n$ to integer (if necessary);

if $(n \geq 0)$ define $x$ to be a tuple with $n$ elements

else define $x$ to be a tuple with 0 elements.

I/O A standard I/O library is available in the system library stdio.pcn. This library deals with formatted $\mathrm{I} / \mathrm{O}$ as in $\mathrm{C}$ but also allows for manipulation of definition variables. 
An Example Using Variable Name '_'

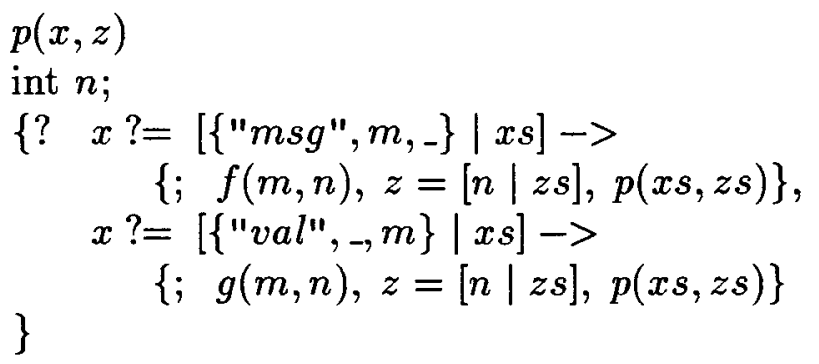

This program has an input argument $x$ and an output argument $z$. The input is a list of 3-element tuples, where the first element of each tuple is the string " $m s g$ " or the string "val". If the first element is the string " $m s g$ " then the second element of the tuple is used by the program to compute $n$ by executing $f(m, n)$, and then $n$ is placed in list $z$. If the second element is the string "val" then the third element of the tuple is used by the program to compute $n$ by executing $g(m, n)$, and then $n$ is placed in list $z$. 


\section{Architectures, Implementation and Effi- ciency}

The speed of execution of a PCN program depends on its implementation. To understand how to develop programs that execute quickly, programmers need to understand something about the implementation of PCN. The implementation may change in future releases, but the central ideas about efficiency are not likely to change significantly.

Uniprocessors, Multiprocessors, and Multicomputers PCN programs run on uniprocessors, multiprocessors or multicomputers. A multiprocessor is a collection of two or more (uni)processors where all processors access a common address space. A multicomputer is a collection of computers where different computers in the collection have disjoint address spaces[10]. A node of a multicomputer (i.e., one of the computers in the collection that forms the multicomputer) can be a uniprocessor, a multiprocessor or a multicomputer. One of the processors in a multiprocessor or a multicomputer is designated the host processor; this is the processor with which programmers interact. (The host processor in a uniprocessor is the uniprocessor itself.) A computer with $n$ processors (in addition to the host) has its processors indexed 0 through $n-1$, the host is indexed $n$. In systems where the host is itself one of the nodes then the host is numbered $n-1$.

Mutables, Definition Variables and Address Spaces A mutable resides in precisely one address space. The implementation does not make copies of mutables. By contrast, a definition variable can have several copies that reside in arbitrarily many address spaces. Since a definition variable is either undefined, or defined and unchanging, all copies of a definition variable are consistent in the following sense: if two copies of a definition variable differ in value then in one of the copies the definition variable is undefined. A program does not make use of undefined definition variables; it merely waits for the definition variable to become defined. Therefore, no problems are created if the value of a definition variable is defined in one copy and undefined in another. 
Consider a block $b$ in a program $p$. If $b$ references mutables (declared in $p$ ) then $b$ is executed in the address space in which $p$ is initiated. This is because there is only one copy of a mutable, and all blocks that access a mutable are executed in the address space in which the mutable resides. If $b$ does not reference mutables then $b$ can be executed in any address space; copies of definition variables are made in the address space in which $b$ is executed, as needed by $b$. The greatest degree of concurrent execution is achieved by employing parallel composition in which the blocks composed in parallel do not share mutables; this allows blocks to be spawned on arbitrary address spaces and thus employs concurrency in multicomputers and multiprocessors.

Granularity If the execution time of a block is small, the time required to spawn the block in a remote address space may exceed the time gained from concurrent execution of the block. Therefore, programmers should ensure that block granularity in parallel composition is appropriate for the target architecture. 


\section{Simple Programming Examples}

\subsection{Membership in a List}

Develop a program member with arguments $x, m$ and $r$, where $x$ is a list, $m$ and $r$ are mutables, and at termination of execution of the program, $r=$ true if and only if $m$ appears in list $x$. Mutable $m$ is to be left unchanged by member. Of course, definition variable $x$ must be left unchanged by member.

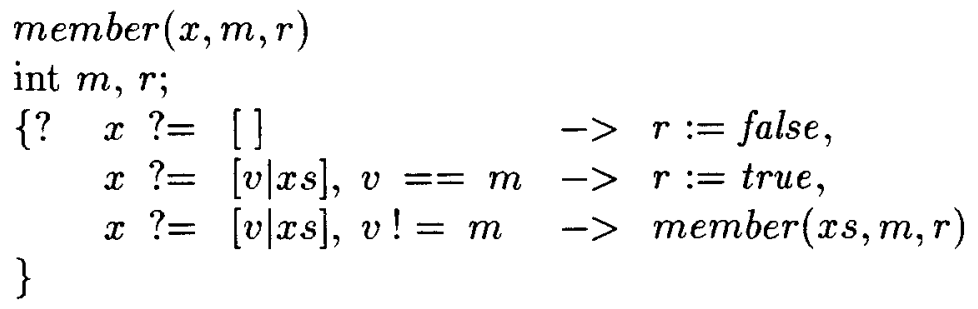

Assume that false $=0$ and true $=1$, to be consistent with C.

\section{Operation of the Program}

1. If $x$ is the empty list, then $r$ becomes false and the program terminates.

2. If $x$ is nonempty, let the head of $x$ be $v$, and let the tail of $x$ be $x s$; if $v=m$ then $r$ becomes true and the program terminates.

3. If $x$ is nonempty and the head of $x$ is not $m$, then the value of $r$ is set by member $(x s, m, r)$, and member $(x, m, r)$ terminates execution when member $(x s, m, r)$ does.

Reasoning About the Program In many examples we reason about the correctness (and the efficiency) of programs by induction. In this example, we carry out induction on the length of $x$. (The length of a list is the number of elements in it.)

Base Case: If $x$ is the empty list then $r$ is false upon termination because $m$ does not appear in an empty list. 
Induction Step: Assume that member $(x, m, r)$ is correct (i.e., it terminates with the correct values of its arguments) for all lists $x$ that have at. most $k$ elements, for some $k \geq 0$, and prove that it is correct for lists with $k+1$ elements. If $x$ has $k+1$ elements it has a head element. Let the head of $x$ be $v$ and let the tail of $x$ be $x s$; then $x s$ has $k$ elements. If $v=m$ then $r$ must be true at termination of the program because $m$ is in list $x$. If $v \neq m$, then at termination $r$ is true if and only if $m$ is in $x s$; by the induction assumption, member $(x s, m, r)$ sets $r$ to true if and only if $m$ is in $x s$.

A Program with Definition Variables Now consider a program with the same specification, except that $m$ and $r$ are definition variables, where $m$ is left unchanged by the program, and $r$ is defined by the program. All we need to do is to remove the declaration of $m$ and $r$, and replace assignment statements by definition statements.

$$
\begin{aligned}
& \text { member } 1(x, m, r)
\end{aligned}
$$

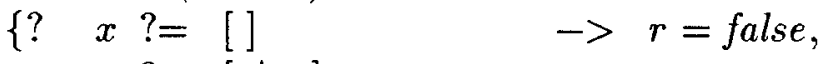

$$
\begin{aligned}
& x ?=[v \mid x s], v==m \rightarrow r=\text { true, } \\
& x ?=[v \mid x s], v !=m \quad \rightarrow \text { member } 1(x s, m, r)
\end{aligned}
$$

\subsection{Sum all Elements in a List}

Develop a program sum with arguments $x$ and $r$, where $x$ is a list of integers and $r$ is a mutable integer. At termination of execution of the program, $r$ is required to be the initial value of $r$ plus the sum of the elements of $x$. List $x$ is to be left unchanged. For example, if $x=[1,2,3]$ and $r=4$ initially, then $r=10$ at termination of the program.

$$
\begin{aligned}
& \operatorname{sum}(x, r) \\
& \text { int } r ; \\
& \{? \quad x \quad ?=[v \mid x s] \quad \rightarrow\{; r:=r+v, \operatorname{sum}(x s, r)\}
\end{aligned}
$$


Operation of the Program In this program:

1. If $x$ is the empty list the program terminates leaving $r$ unchanged.

2. If $x$ is nonempty, let $v$ be the head of $x$ and let $x s$ be the tail of $x$; mutable $r$ becomes $r+v$, and then $\operatorname{sum}(x s, r)$ is executed.

Reasoning About the Program Let $r^{i}$ be the initial value of $r$, and let $r^{f}$ be the value of $r$ when program sum terminates execution. We reason about the program by inducting on the length of $x$.

Base Case: If $x$ is the empty list, the program terminates and $r$ is left unmodified, and hence $r^{f}=r^{i}$, as required.

Induction Step: Assume that program $\operatorname{sum}(x, r)$ is correct for all lists $x$ with length at most $k$, for some $k \geq 0$, and prove that it is correct for lists $x$ with length $k+1$. If the length of $x$ is $k+1$ then $x$ is nonempty; let $v$ be the head of $x$ and let $x s$ be the tail of $x$. List $x s$ has length $k$. From the induction assumption, $\operatorname{sum}(x s, r)$ is correct. Hence $r^{f}=r^{i}+v+$ sum of elements in $x s$, and hence $r^{f}$ is the sum of $r^{i}$ and the sum of all elements of $x$, as required by the specification.

Another Summation Example Next we write a program total to define a definition variable $z$ as the sum of all the elements of a list $x$. The difference between this program and the previous one is that $z$ is definition variable whereas $r$ is mutable, and furthermore $z$ is to be the sum of the elements of $x$, whereas the sum of the elements of $x$ was added to $r$ in the previous program.

$\operatorname{total}(x, z)$

int $r$;

$\{; \quad r:=0, \operatorname{sum}(x, r), z=r\}$

An alternate version total1, using only definition variables and parallel composition, is given next. 


$$
\begin{aligned}
& \text { total1 }(x, z) \\
& \{? \quad x ?=[] \quad \rightarrow z=0 \text {, } \\
& x ?=[v \mid x s] \quad \rightarrow\{\| z=z s+v, \operatorname{total} 1(x s, z s)\} \\
& \text { \} }
\end{aligned}
$$

Operation of the Program The operation of this program is as follows.

1. If $x$ is the empty list then definition variable $z$ is defined to be 0 .

2. If $x$ is nonempty, let $v$ be the head of $x$ and let $x s$ be the tail of $x$. Define definition variable $z$ to be the expression $z s+v$, where $z s$ is defined by total1 $(x s, z s)$.

Reasoning About the Program We are obliged to ensure that blocks in parallel composition do not modify shared mutables. This program has no mutables, and so the restriction about shared variables holds vacuously.

We reason by induction on the length of $x$, as in the previous example. The reasoning is not given here because it is largely a repetition of the arguments given earlier.

Difference Between Programs Let $x$ be the list $[1,2]$. At the termination of $\operatorname{total}(x, z)$, definition variable $z$ is defined to be the number 3 . At the termination of $\operatorname{total} 1(x, z)$, definition variable $z$ is defined by the following equations:

$z=1+a$, where $a$ is defined by

$a=2+b$, where $b$ is defined by

$b=0$.

If we now execute $m:=z$, where $m$ is a mutable, and then print $m$, we will get the same answer whether we use total or total 1 because the reduced value of $z$ is the same in both cases.

At first glance, sequential programs such as total may appear more efficient in memory and time than parallel programs such as totall. Consider the following variation in which a parallel implementation can require less time than a sequential implementation. 


\subsection{Sum Function of Elements in a List}

Let $x$ be a list. Define $z$ as follows: $z$ is the sum over all elements of the list of a function $g$ applied to each element. For example, if $x$ is $[1,2]$ and $g$ is the square operation, then $z=5$.

A sequential program sigma, analogous to sum in the previous subsection, is given next.

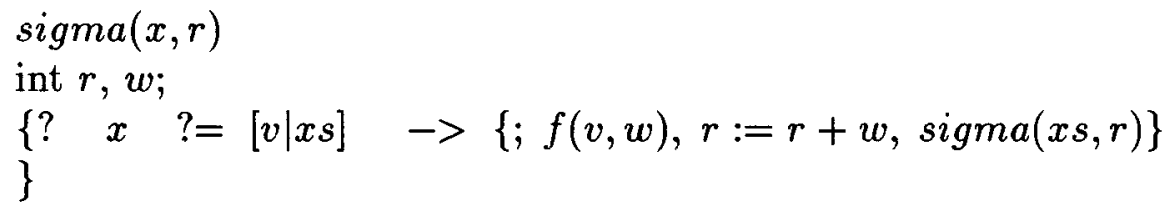

Program $f(v, w)$ sets the value of $w$ to be $g(v)$.

Program Operation The operation of this program is as follows. If $x$ is empty the program terminates with $r$ unchanged. If $x$ is not empty, let $v$ be the head of $x$, and let $x s$ be the tail of $x$; first compute $w$, then increment $r$ and then call sigma(xs,r) to sum the remainder of the list.

Program tote, given next, is analogous to total of the previous subsection.

tote $(x, z)$

int $r$;

$\{; \quad r:=0, \operatorname{sigma}(x, r), z=r\}$

A version using parallel composition is given next.

$\operatorname{totpar}(x, z)$

int $w$;

$\{? \quad x ?=[] \quad \rightarrow z=0$,

$x ?=[v \mid x s] \quad \rightarrow\{\|\{; f(v, w), y=w\}$,

$z=z s+y$,

totpar $(x s, z s)$

\}

\} 
This program spawns programs $f(v, w)$, for each element $v$ of list $x$, in parallel. Thus if $f$ takes a long time to execute, and $x$ is a large list, and there are a large number of processors, the parallel version will execute faster than the sequential version, because execution of $f$ for different elements of the list will proceed in parallel.

In program totpar we are obliged to ensure that no mutable shared variable in a parallel composition block is modified. There are no mutable shared variables in the parallel composition block, and hence the restriction is satisfied.

Consider an erroneous program in which the parallel composition block of totpar is replaced:

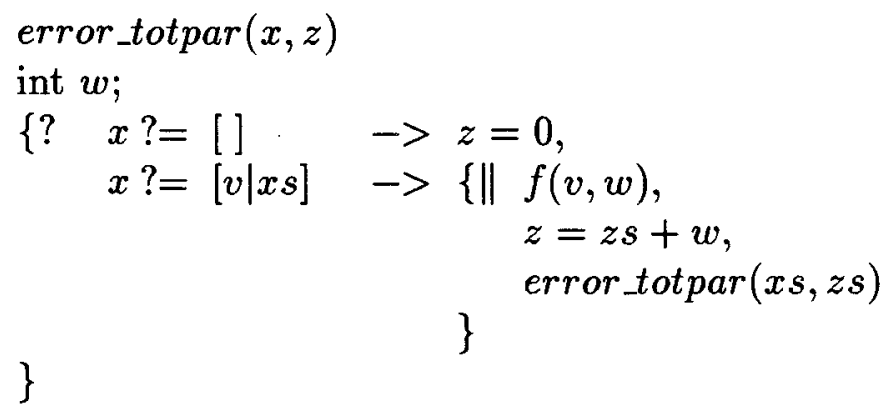

In this program, $w$ is a mutable shared by blocks $f(v, w)$ and $z=z s+w$ in a parallel composition block, and $w$ is modified by $f(v, w)$; this violates the restriction on shared mutables in parallel composition. The reason for the restriction is that program error_totpar $(x, z)$ does not specify what value of $w$ is to be used in the definition $z=z s+w$ : is it the value of $w$ before, during or after the execution of $f(v, w)$ ? This problem does not arise in totpar because the shared definition variable $y$ is used in place of shared mutable $w$, and $y$ is defined as the correct value of $w$ - the value of $w$ after $f(v, w)$ is executed by means of the sequential composition block $\{; f(v, w), y=w\}$.

\subsection{Reverse a List}

Develop a program $r e v$ with arguments $x, e$ and $b$, where $x$ and $e$ are lists that are to be left unchanged by $r e v$, and $b$ is to be defined by $r e v$ to be the list of elements in $x$, in reverse order, concatenated with $e$. For example, if $x=$ $[" A ", " B "]$, and $e=[" C ", " D "]$, then $b$ is to be defined as [" $B ", " A ", " C ", " D "]$. 
(The name $b$ stands for the beginning of the reversed list, and $e$ stands for the end of the reversed list.)

$$
\begin{aligned}
& r e v(x, e, b)
\end{aligned}
$$

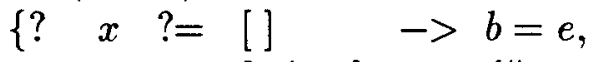

$$
\begin{aligned}
& x ?=[v \mid x s] \rightarrow\{\| e s=[v \mid e], \operatorname{rev}(x s, e s, b)\}
\end{aligned}
$$

\section{Operation of the Program}

1. If $x$ is the empty list, then $b=e$.

2. If $x$ is nonempty, let $v$ be the head of $x$ and let $x s$ be the tail of $x$. Define $b$ by $\operatorname{rev}(x s, e s, b)$, where $e s$ is defined as $v$ followed by $e$. For example, if $x=\left[" A^{\prime \prime}, B^{\prime \prime}\right]$, and $e=\left[" C^{\prime \prime}, " D^{\prime \prime}\right]$, then $v$ is " $A^{\prime \prime}$, and $x s$ is [" $\left.B^{\prime \prime}\right]$. Hence, $e s$ is [" $A ", " C ", " D "]$.

Reasoning About the Program We first ensure that mutable shared variables are not modified in parallel composition, and then reason about the program by induction on the length of $x$. This reasoning is very similar to that given for the previous programs, and is left to the reader.

\subsection{Height Of A Binary Tree}

Develop a program $h t$ with arguments $t$ and $z$, where $t$ is a binary tree, and $z$ is to be defined to be the height of the tree. A tree $t$ is either the empty tuple, \{\} , or a 3-tuple \{left,val, right $\}$, where left and right are the left and right subtrees of $t$. Both $t$ and $z$ are definition variables, and $t$ is to be left unchanged by the program.

$$
\begin{aligned}
& h t(t, z) \\
& \{? \quad t ?=\{\} \quad \rightarrow z=0, \\
& t ?=\{\text { left, val,right }\} \quad \rightarrow\{\| h t(\text { left }, \text { l), ht }(\text { right }, r), \\
& \{? l>=r->z=1+l \text {, } \\
& r>=l \rightarrow z=1+r \\
& \text { \} }
\end{aligned}
$$




\section{Operation of the Program}

1. If $t$ is the empty tuple, in which case $t$ is the empty tree, its height $z$ is defined to be 0 .

2. If $t$ is not the empty tree, then $t$ is a 3-tuple. Let $t$ be the tuple $\{l e f t, v a l, r i g h t\}$. Define definition variables $l$ and $r$ by $h t(l e f t, l)$ and $h t(r i g h t, r)$, respectively. If $l$ exceeds $r$ then define $z$ as the expression $1+l$. If $r$ exceeds $l$ then define $z$ as the expression $1+r$. If $r=l$, then define $z$ either as $1+l$ or $1+r$.

Reasoning About the Program We check that mutable shared variables are not modified in parallel composition. We reason about the program by induction on the height of tree $t$.

Base Case: If $t$ is the empty tree, then its height $z$ is defined (correctly) as 0 .

Induction Step: Assume that the program is correct for all trees $t$ with height at most $k$ for some $k \geq 0$, and prove that the program is correct for all trees with height $k+1$. If $t$ is a tree with height $k+1$, then $t$ is a tuple of the form \{left,val,right $\}$ where the heights of trees left and right are at most $k$. By the induction assumption, $h t(l e f t, l)$ and $h t(r i g h t, r)$ correctly define $l$ and $r$ to be the heights of the left and right subtrees of $t$. Hence $z$ is 1 more than the height of the higher subtree.

\subsection{Preorder Traversal of a Binary Tree}

Develop a program preorder with arguments $t, b$ and $e$, where $t$ is a binary tree, $b$ and $e$ are lists. Binary trees are represented using tuples as in the last example. Parameters $t$ and $e$ are to be left unchanged by the program. List $b$ is to be the list consisting of the $v a l$ of all nodes of the tree in preorder, 
concatenated with list $e$. (A traversal of a tree in preorder visits the root, then the left subtree, and finally the right subtree.)

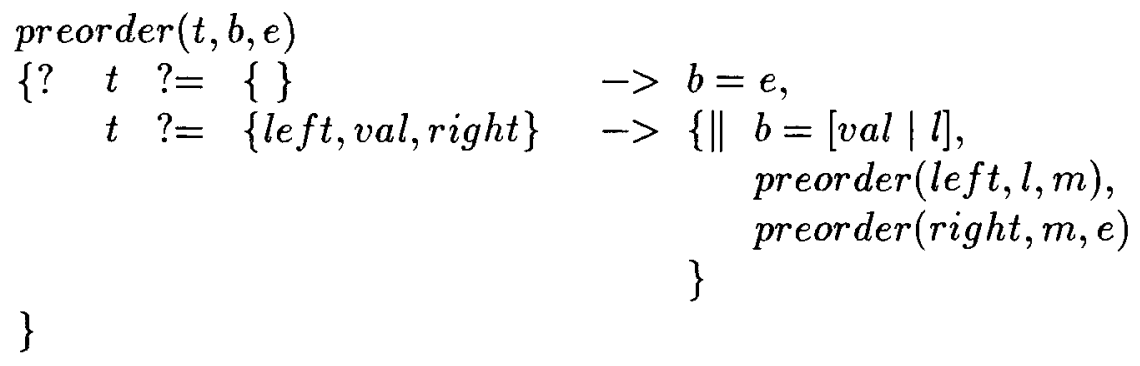

\}

\section{Operation of the Program}

1. If $t$ is the empty tree then $b$ is defined as $e$.

2. If $t$ is not empty, then it is a tuple of the form $\{l e f t, v a l$,right $\}$. In parallel, define $m$ by preorder (right, $m, e)$, and $l$ by preorder $(l e f t, l, m)$, and $b$ as val followed by $l$.

Reasoning About the Program First check that shared mutables are not modified in parallel composition.

We prove correctness of the program by induction on the height of tree $t$.

Base Case: If $t$ is the empty tree, then $b=e$, because there are no nodes to traverse.

Induction Step: Assume that preorder $(t, b, e)$ is correct for trees of height at most $k, k \geq 0$, and show that it is also correct for trees of height $k+1$. Let $t$ be a tree of height $k+1$. Then $t=\{l e f t, v a l$, right $\}$, where left and right are trees of height at most $k$. Define a definition variable $m$ by preorder (right, $m, e)$. By the induction assumption, $m$ is the preorder traversal of the right subtree concatenated with $e$. Define a definition variable $l$ by preorder $(l e f t, l, m)$. By the induction assumption, $l$ is the preorder traversal of the left subtree concatenated with $m$. Define $b$ as $[\mathrm{val} \mid \mathrm{l}]$; hence, $b$ is val 
followed by the preorder traversal of the left subtree of $t$ followed by the preorder traversal of the right subtree of $t$, and hence $b$ is the preorder traversal of $t$.

\subsection{Quicksort with Copying}

In this section we present C.A.R.Hoare's quicksort [9] program, $q 0$, that uses lists (of definition variables); later, we discuss an in place quicksort, $q 1$, that uses arrays. (The quicksort algorithm is discussed in most texts on algorithms such as $[1]$.

In this section, when we refer to a list of numbers we mean a list of definition variables that (eventually) reduce to numbers. Program $q 0$ has two input variables, $x$ and end, and one output variable, $z$ : variables $x$ and end are definition variables that are not defined by the program, and $z$ is a definition variable that is defined by the program. All three variables are lists of numbers. The output $z$ is specified to be the list $x$ sorted in increasing order concatenated with list end. For example if $e n d=[5,4]$ and $x=[2,1]$, then $z=[1,2,5,4]$. If end is the empty list, then $z$ is $x$ sorted in increasing order.

$$
\begin{aligned}
& q 0(x, e n d, z)
\end{aligned}
$$

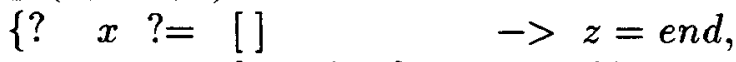

$$
\begin{aligned}
& x ?=[\operatorname{mid} \mid x s] \quad \rightarrow\{\| \operatorname{part}(\text { mid, } x \text { s, left,right }), \\
& q 0(\text { left },[\text { mid } \mid r], z) \text {, } \\
& q 0(\text { right }, \text { end }, r) \\
& \text { \} }
\end{aligned}
$$

Operation of the Program The operation of program $q 0$ is as follows. If $x$ is the empty list then $z$ is defined to be end. If $x$ is nonempty, let mid be the first element of $x$, and let $x s$ be the remaining elements of $x$. The call part (mid, xs, left, right) defines left to be the list of values of $x s$ that are at most mid, and right to be the list of values of $x s$ that exceed mid. Call $q 0$ (right, end, $r$ ), thus defining $r$ to be the sorted list of right appended to end. Call $q 0($ left, $[$ mid $\mid r], z)$, thus defining $z$ to be the sorted list of left followed by mid followed by $r$. 
Reasoning About the Program There are no shared mutables in the parallel composition, and hence the restrictions on parallel composition are satisfied. We reason about this program by induction on the length of $x$. The reasoning is straightforward and is left to the reader.

Next, we discuss program part. Program part inspects each element of $x s$ in turn, placing elements that are at most mid in left and all other elements in right.

$$
\begin{aligned}
& \text { part (mid, xs, left, right) }
\end{aligned}
$$

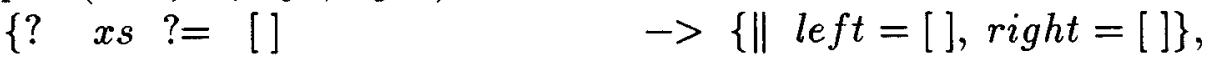

$$
\begin{aligned}
& x s ?=[h d \mid t l], h d<=\operatorname{mid} \rightarrow\{\{\| \text { left }=[h d \mid l s] \text {, } \\
& \operatorname{part}(\text { mid }, t l, l s, \text { right }) \\
& x s ?=[h d \mid t l], h d>\operatorname{mid} \rightarrow\{\| \text { right }=[h d \mid r s], \\
& \operatorname{part}(\text { mid, } t l, \text { left, rs })
\end{aligned}
$$

Operation of the Program If $x s$ is the empty list, define left and right to be empty lists. If $x s$ is not empty, then let $h d$ and $t l$ be the head and tail (respectively) of $x s$. If $h d$ is at most mid, define $l s$ and right by $p a r t(m i d, t l, l s, r i g h t)$, and define left as $h d$ followed by $l s$. If $h d$ exceeds mid, define left and $r s$ by $\operatorname{part}(\operatorname{mid}, t l, l e f t, r s)$, and define right as $h d$ followed by $r s$.

Reasoning About the Program We check that shared mutables are not modified in parallel composition, and reason about the program by induction on the length of $x s$.

Base Case: If $x s$ is the empty list, then left and right are correctly defined as empty lists.

Induction Step: Assume that the program is correct for all mid, $x s$, where the length of $x s$ is at most $k$, for some $k \geq 0$, and prove that the program is correct for all $x s$ of length $k+1$. If the length of $x s$ is $k+1$, then 
$x s$ is nonempty; let $h d$ and $t l$ be the head and tail (respectively) of $x s$. The length of $t l$ is $k$. Next, consider the case where $h d$ is at most mid. From the induction assumption, part(mid, $t l, l s$, right) defines $l s$ and right to be the elements of $t l$ that are at most mid, and that exceed mid, respectively. Since, $x s$ is $h d$ followed by $t l$, and $h d \leq m i d$, it follows that the sequence of elements of $x s$ that are at most mid is $h d$ followed by the sequence of elements of $t l$ that are at most mid, and the sequence of elements of $x s$ that exceed mid is the sequence of elements of $t l$ that exceed mid. Hence, in this case, the definitions of left and right are correct. A similar argument applies for the case where $h d>$ mid.

\subsection{In Place Quicksort}

Program $q 1$ Program $q 1$ has two input parameters, $l$, and $r$, both of which are definition variables, and it has one input-output parameter $C$ which is a one-dimensional array of numbers. Let $C^{\text {init }}$ be the initial value of $C$, and let $C^{f i n a l}$ be the value of $C$ on termination of the program. Then $C^{f i n a l}$ is to be a permutation of $C^{i n i t}$, where $C^{f i n a l}[l, \ldots, r]$ is $C^{i n i t}[l, \ldots, r]$ in increasing order, and the other elements of $C$ are to remain unchanged. (If $l \geq r$ then $C^{f i n a l}$ is $C^{\text {init }}$.)

$q 1(l, r, C)$

int $C[]$;

$\{? l<r \rightarrow\{;$ split $(l, r, C$, mid $)$,

$\{\| q 1(l, \operatorname{mid}-1, C), q 1(\operatorname{mid}+1, r, C)\}$

\}

\}

Execution of split $(l, r, C$, mid $)$ permutes $C$ and assigns a value to mid such that $l \leq m i d \leq r$, and such that all elements in $C[l, \ldots$, mid -1$]$ are at most $C[$ mid $]$, and all elements in $C[$ mid $+1, \ldots, r]$ exceed $C[$ mid $]$.

Operation of the Program If $l \geq r$, then $q 1$ takes no action, leaving $C$ unchanged. If $l<r$, then split is called, and after split terminates execution, $C[l, \ldots$, mid -1$]$ and $C[$ mid $+1, \ldots, r]$ are sorted in parallel. 
Reasoning About the Program First check that shared mutables in parallel composition are not modified. Array $C$ is shared by $q 1(l$, mid $-1, C)$ and $q 1($ mid $+1, r, C)$, but no element of $C$ is shared by both blocks. Hence the restriction on parallel composition is satisfied.

We reason about the program by induction on $r-l$.

Base Case: If $r-l \leq 0$, then $C$ is left unchanged, and this is correct according to our specifications.

Induction Step: Assume that $q 1(l, r, C)$ is correct for all $l, r$, and $C$ for which $r-l \leq k$, for some $k \geq 0$, and prove that the program is correct for $r-l=k+1$. If $r-l=k+1$ then $l<r$. In this case split is called, and execution of split $(l, r, C$, mid $)$ permutes $C$ and assigns a value to mid such that $l \leq$ mid $\leq r$, and such that all elements in $C[l, \ldots$, mid -1$]$ are at most $C[m i d]$, and all elements in $C[$ mid $+1, \ldots, r]$ exceed $C[m i d]$. Hence, mid $-1-l \leq k$, and $r-($ mid +1$) \leq k$. From the induction assumption, $q 1(l$, mid $-1, C)$ and $q 1($ mid $+1, r, C)$ are correct, and sort $C[l, \ldots$, mid -1$]$ and $C[$ mid $+1, \ldots, r]$. Therefore, $C[l, \ldots, r]$ is sorted correctly.

\section{Program split}

$\operatorname{split}(l, r, C$, mid $)$

int $C[]$, left, right, temp;

$\{? l<=r \rightarrow$

$\{; \quad$ left $:=l+1$, right $:=r, s=C[l]$, part1 $(l, r, C, s$, left, right $)$, temp $:=l$, swap $($ temp, right,$C)$, mid $=$ right

\}

\}

Operation of the Program If $l>r$ then split terminates execution without taking any action. If $l \leq r$, then program split $(l, r, C$, mid $)$ calls

part1 $(l, r, C, s$, left, right $)$ after setting left $=l+1$, right $=r$ and $s=C[l]$; program part leaves $s$ unchanged, modifies left and right, and permutes elements of $C[l+1, \ldots, r]$ so that, at termination of part 1 , left $=$ right +1 , 
and all elements in $C[l+1, \ldots, r i g h t]$ are at most $s$, and all elements in $C[$ right $+1, \ldots, r]$ exceed $s$.

After termination of part1, program swap is called to exchange $C[l]$ (which is $s$ ) with $C[$ right $]$. After the swap, all elements in $C[l, \ldots, r i g h t-1]$ are at most $s$, and $C[r i g h t]=s$, and all elements in $C[$ right $+1, \ldots, r]$ exceed $s$. The program terminates after mid is defined as right.

Program part1 Program part 1 moves left rightwards and moves right leftwards until they cross (i.e., left $=$ right +1 ), so that the following invariant is maintained:

\section{invariant:}

$l+1 \leq$ left $\leq r+1$ and $l \leq$ right $\leq r$, and all elements of $C[l+1, \ldots$, left -1$]$ are at most $s$, and all elements of $C[r i g h t+1, \ldots, r]$ exceed $s$.

From the invariant it follows that left $\leq$ right +1 .

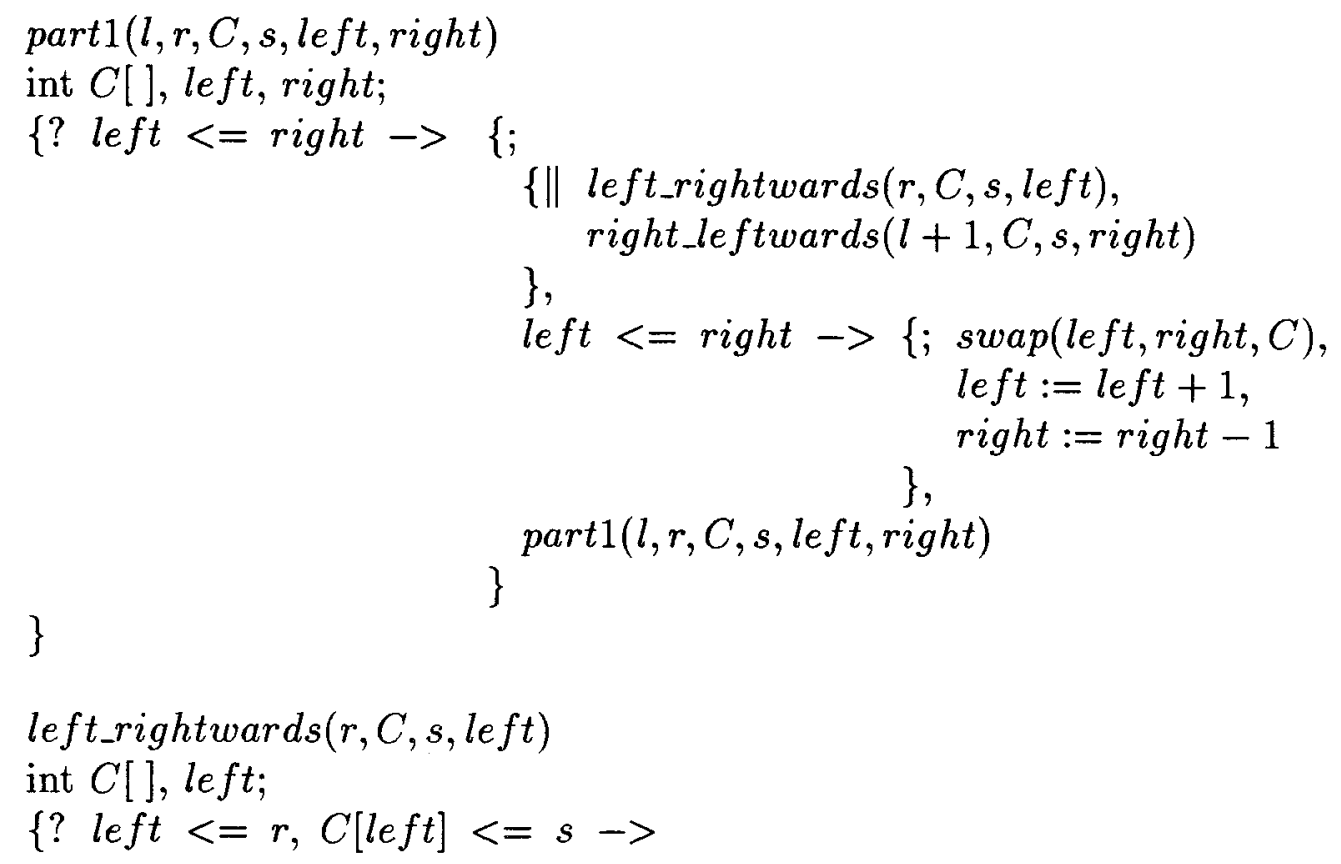




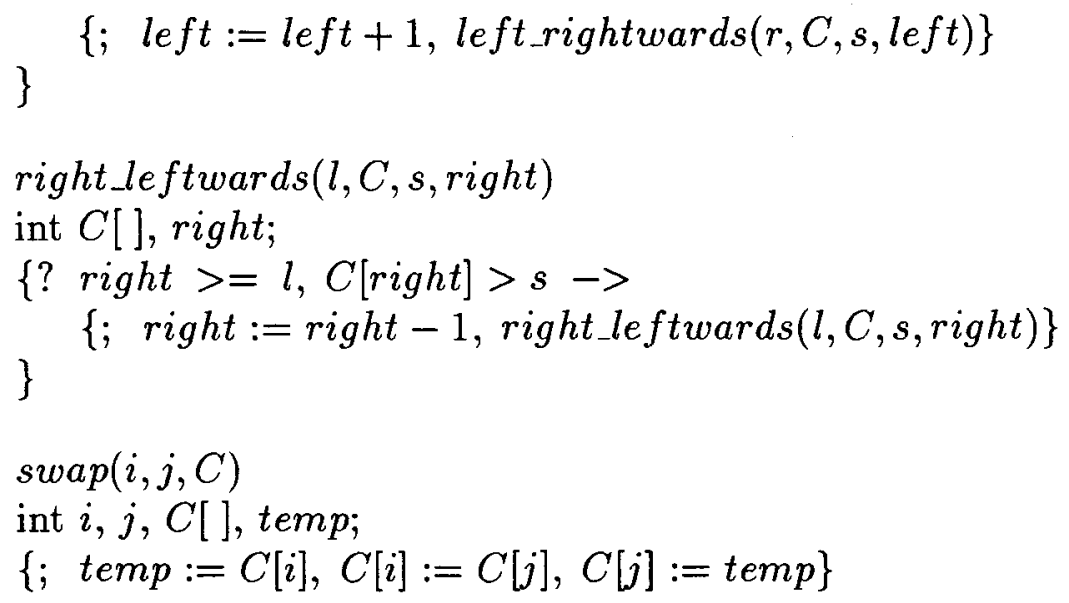

Operation of the Program The invariant holds initially because le ft $=$ $l+1$ and right $=r$. Programs left_rightwards $(r, C, s, l e f t)$ and

right_leftwards $(l+1, C, s$, right $)$ are executed in parallel. The only mutables that change value in these programs are left and right, and these mutables are not shared. Therefore, the restriction on parallel composition is satisfied.

Program left_rightwards $(r, C, s$, left $)$ moves left rightwards from $l+1$, i.e., it increases left, until left $=r+1$ or $C[l e f t]>s$, and it maintains the invariant. Likewise, right_leftwards $(l+1, C, s$, right $)$ moves right leftwards from $r$, i.e., it decreases right, until right $=l$ or $C[$ right $] \leq s$, and it maintains the invariant. If left $=r+1$ or right $=l$ then left $>$ right, and the program terminates execution. Consider the case where $C[$ right $] \leq s<C[$ left], and left $\leq$ right at termination of the parallel composition block. In this case, $C[l e f t]$ and $C[$ right $]$ are exchanged, and then left is incremented and right is decremented, maintaining the invariant. Then part1 is calls itself.

Reasoning About the Program We reason about the program by induction on right $+1-$ left.

Base Case: Consider the case where right $+1-$ left $=0$. In this case the program terminates. If the invariant holds, the program terminates correctly. (The program may not terminate correctly if the invariant does not hold.) 
Induction Step: Assume that the program is correct, for all parameters provided right $+1-$ left $\leq k$, for some $k \geq 0$, provided the invariant holds when the program is initiated, and prove the program correct for right $+1-$ left $=$ $k+1$ provided the invariant holds when the program is initiated.

If right $+1-$ left $=k+1$ then left $\leq$ right. In this case the parallel composition of right_leftwards and left_rightwards maintains the invariant and decreases right $+1-l e f t$, or it leaves left and right unchanged. In the latter case, $C[$ right $] \leq s<C[l e f t]$, and therefore, $C[l$ left $]$ and $C[$ right $]$ are exchanged, and then left is incremented and right is decremented, maintaining the invariant, and reducing right $+1-$ left. Therefore, when part 1 is called recursively, the invariant is maintained, and right $+1-l e f t \leq k$. From the induction assumption, the recursive call to part 1 terminates execution with the correct values for $C$, left and right. 


\section{Instructions About Where to Execute Pro- grams}

For purposes of efficiency, programmers may want to specify the processors on which programs are to be executed. For this purpose the annotation '@' is added as a suffix to the program call [6]. Specifying processors does not change the semantics of programs.

The syntax of an annotated program call is:

program-call :: program-call@location

location $\quad::$ variable | integer | relative-location

A PCN program runs on a computer in which processors are numbered $0 \ldots n$, where 0 is the host machine. A program $p$ can be executed on a processor numbered $i$, for any integer $i$ where $0<i \leq n$ by executing $p @ i$ or $p @ v$, where $v$ is a variable with reduced value $i$.

PCN can execute on a network of processors where the topology of the network can take any form: It can be a local area network connecting workstations, or a hypercube, or a mesh, to name a few examples. Programmers find it convenient to develop their programs for a given topology, and to have their topology mapped to that of the machine on which their programs execute $[6]$.

The processor in the virtual network at which a program is to be executed can be specified by appending orelative-location to the program call. For example, on a ring:

relative-location can be fwd for forward, bwd for backward, or random;

The relative-location specifies a processor in the virtual network by specifying its relationship (forward, backward, or random) with respect to the 
processor executing the parallel composition block. For instance, execution of a parallel composition statement $\{\| \quad p($ left $) @ b w d, p($ right $) @ f w d\}$ in a processor $q$ in a virtual ring would cause $p(l e f t)$ and $p(r i g h t)$ to be executed on the processor following $q$ and preceding $q$, respectively, on the virtual ring.

The location at which a program $p(.$.$) is to be executed can be specified$ at run-time by executing the statement $p\left(. . . @^{\prime} x\right.$, where $x$ is a definition variable. Execution of $p(\ldots) @^{\prime} x$, is as follows:

repeat skip until $x$ is reducible; execute $p @ l o c$ where "loc" is the reduced value of $x$.

For instance, if the reduced value of $x$ is " $f w d^{\prime \prime}$, then $p(\ldots) @^{\prime} x$ is executed as $p(. .) @ f w$.$d .$

If a program call is executed in an address space then the called program will not be executed in another address space if any argument of the called program is a mutable. 


\section{Compilation and Modules}

Collections of related PCN programs are written in files, for convenience. All PCN programs can be put in a single file, but it is usually more convenient to partition programs in some logical manner, and put each set of related programs in a separate file [6]. A file containing PCN programs is called a module, and the name of the file is also the module name. The syntax of a module is:

$$
\begin{aligned}
\text { module:: } & - \text { exports }\left(\prec \text { program-name } \succ^{(1)}\right) \\
& - \text { foreign }\left(\prec \text { library-name } \succ^{(1)}\right) \\
& \prec \text { program } \succ
\end{aligned}
$$

where program-name is the name of one of the programs in the module, and library-name is the name of a library containing $\mathrm{C}$ object code, and all names are quoted.

An example of a module is:

$$
\begin{aligned}
& \text {-exports ("member", "sum") } \\
& \text {-foreign("algebra.a", "diffeqns.o") } \\
& \text {... definition of programs including member and sum ... }
\end{aligned}
$$

If there is no export statement in a module then all programs are exported. A program $p$ within a module $m$ can be called by programs in other modules if and only if $p$ is exported by $m$. A program in a module references a PCN program in another module by prefixing the name of the referenced program 
with the name of the module in which the referenced program is located, followed by the symbol ' $\because$ '. For instance, a program in a module $B$ references a program $p$ in another module $D$ as $D: p$. A program references another program in the same module without the 'module-name:' prefix.

PCN programs can also call $\mathrm{C}$ and Fortran programs. Consider a PCN program $p$ that calls a $\mathrm{C}$ program $f$ that appears in a library $L$. The module in which $p$ appears must have library $L$ declared as foreign, by including $L$ in the -foreign( $\prec$ library-name $\succ$ ) statement that appears in the module.

A PCN source file can begin with macro definitions and file inclusion statements as in $\mathrm{C}$. 


\section{Acknowledgment}

The PCN project is a team effort. Institutions that have participated in the effort include the Center for Research in Parallel Computation, California Institute of Technology, Argonne National Laboratories and Aerospace Corporation. Enhancements to $\mathrm{PCN}$ are planned with participation from Rice University and Los Alamos National Laboratories.

Scientists who have contributed to the PCN project include Sharon Brunett, Jan Lindheim, Dave Long, Dong Lin, Berna Massengill and Seth Noble at Caltech, Ian Foster and Steve Tuecke at Argonne, and Joe Bannister, A. Campbell, Ray Chowkwanyun, Mel Cutler, Melody Hancock, Carl Kesselman, Craig Lee at Aerospace Corp. The run-time system was developed at Argonne by Ian Foster and Steve Tuecke. A programming environment with a variety of support tools is being developed at Aerospace Corp. The development of PCN has been aided by applications written in PCN for weather modeling and DNA sequence matching at Argonne, computing trajectories of space vehicles and tracking objects in space at Aerospace, and Taylor-Couette flows at Caltech. We are grateful to scientists who have given of their time in helping develop PCN applications, particularly to Herb Keller at Caltech.

We are grateful to our sponsors at the National Science Foundation, the Air Force Office of Scientific Research and the Office of Naval Research for their support and advice; in particular, we wish to thank Nat Macon, Harry Hedges, Charles Holland and Andre van Tilborg.

Many ideas in PCN are derived from ideas in UNITY and Strand. 


\section{References}

[1] Aho, A.V., J. E. Hopcroft, and J. D. Ullman, Addison-Wesley, Data Structures and Algorithms, Reading, Massachusetts.

[2] Campbell, A., R.M. Chowkwanyun, C.F.Kesselman, C. A. Lee, and S. Taylor, 'A Database System to Support the Program Composition Environment', Aerospace Corp., Report No. TOR-0090(5920-05)-1, April 1990.

[3] Chandy, K. M., and J. Misra, Parallel Program Design: A Foundation, Addison-Wesley, Reading, Massachusetts, 1988.

[4] Chandy,K.M., and S.Taylor, 'The Composition of Concurrent Programs,' in Proceedings Supercomputing '89, Reno, Nevada, Nov.13-17, 1989, ACM.

[5] Dijkstra, E. W., A Discipline of Programming, Prentice-Hall, Englewood Cliffs, New Jersey, 1976.

[6] Foster,I., and S.Taylor, Strand, New Concepts in Parallel Programming, Prentice-Hall, 1989.

[7] Foster,I., and S.Taylor, 'A Portable Run-Time System for PCN', Argonne National Laboratory Report No. ANL/MCS-TM-137, January 1990.

[8] Hoare, C.A.R., Communication Sequential Processes, Prentice-Hall International, London, U.K., 1984.

[9] Hoare, C.A.R., 'Quicksort,' Computer J., Vol.5, No.1, pp10-15, 1962.

[10] Seitz, C.L., and J. Seizovic, and W. Su, 'The C Programmer's Abbreviated Guide to Multicomputer Programming', Caltech Computer Science Technical Report Caltech-CS-TR-88-1, 19 January 1988, revised 17 April 1989. 


\section{A Complete PCN Syntax}

Non-terminal symbols begin with an uppercase letter. Token classes such as an integer, identifier (ID), or string are signified in uppercase and are lexically identical to C. All other symbols are terminal.

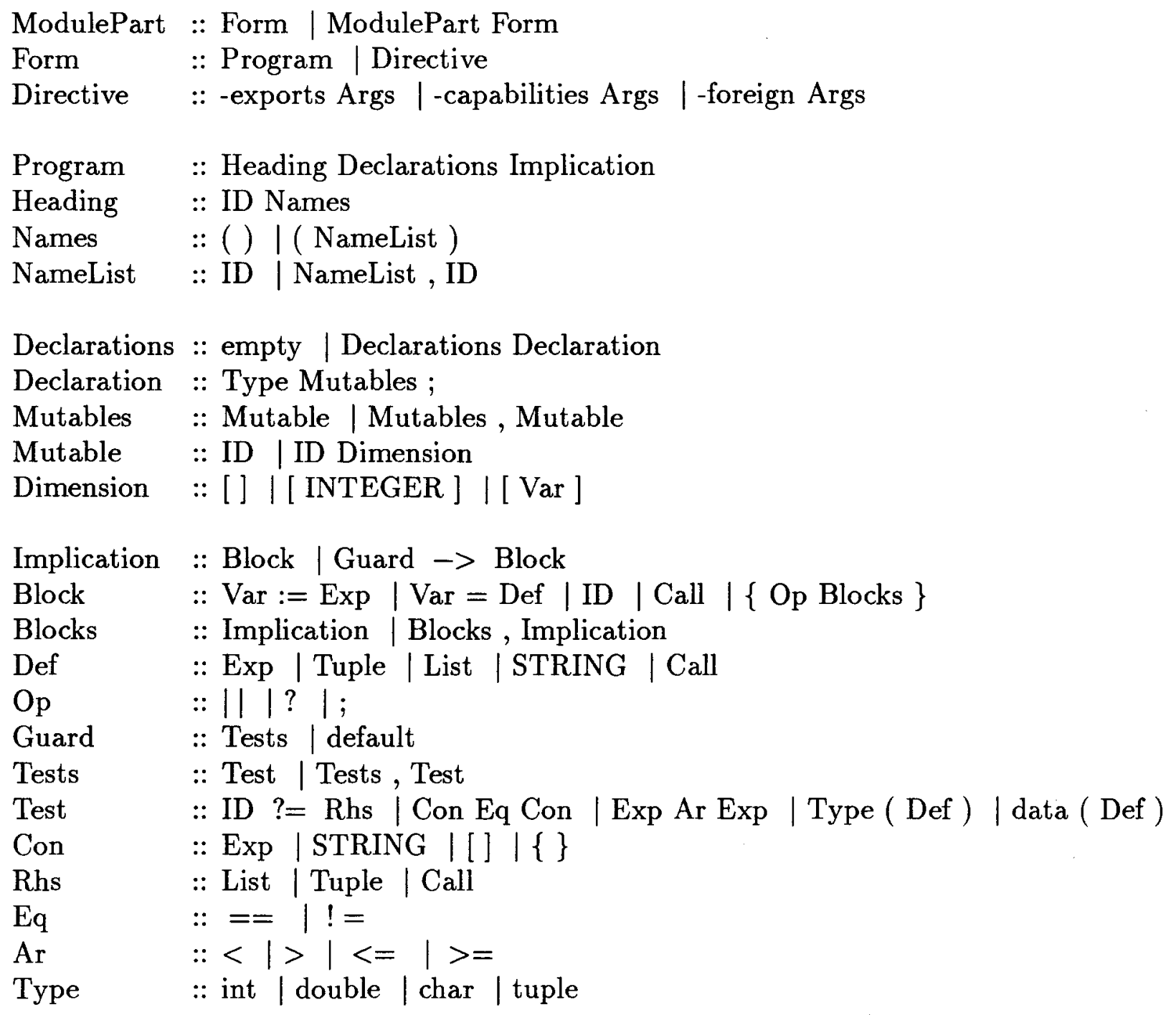




\begin{tabular}{|c|c|}
\hline Call & :: LocalCall | RemoteCall \\
\hline LocalCall & $\because:$ QID : QID Args | QID Args \\
\hline QID & $::$ ID |' ID \\
\hline RemoteCall & :: LocalCall @ INTEGER | LocalCall @ QID \\
\hline Args & $::() \mid($ ArgList $)$ \\
\hline ArgList & $::$ Def $\mid$ ArgList, Def \\
\hline $\operatorname{Exp}$ & $::$ Term | Exp + Term $\mid \operatorname{Exp}-$ Term \\
\hline Term & $::$ Factor | Term $*$ Factor | Term Factor | Term \% Factor \\
\hline Factor & $::$ Num | ( Exp ) | length (ID ) \\
\hline Num & $::$ Numeric | - Numeric | Var \\
\hline Numeric & $::$ INTEGER | REAL \\
\hline Var & $::$ ID | ID Subscript \\
\hline Subscript & $::[$ INTEGER ] | [ Var ] \\
\hline List & $::[] \mid[$ Elements ] | [ Elements | Element ] \\
\hline Tuple & $::\{$ Elements $\} \mid\{\}$ \\
\hline Elements & $::$ Element | Elements, Element \\
\hline Element & :: Num | STRING | List | Tuple | Call \\
\hline
\end{tabular}

